\title{
Positive selection on human gamete-recognition genes
}

\author{
Michael W Hart ${ }^{\text {Corresp., }}{ }^{1}$, Daryn A Stover ${ }^{2}$, Vanessa Guerra ${ }^{1}$, Sahar V Mozaffari ${ }^{3}$, Carole Ober $^{3}$, Carina F \\ Mugal $^{4}$, Ingemar Kaj ${ }^{5}$ \\ 1 Department of Biological Sciences, Simon Fraser University, Burnaby, British Columbia, Canada \\ 2 School of Mathematical and Natural Sciences, Arizona State University Colleges at Lake Havasu City, Lake Havasu City, Arizona, USA \\ 3 Department of Human Genetics, University of Chicago, Chicago, Illinois, USA \\ 4 Department of Ecology and Genetics, Uppsala University, Uppsala, Sweden \\ 5 Department of Mathematics, Uppsala University, Uppsala, Sweden \\ Corresponding Author: Michael W Hart \\ Email address: mwhart@sfu.ca
}

Coevolution of genes that encode interacting proteins expressed on the surfaces of sperm and eggs can lead to variation in reproductive compatibility between mates and reproductive isolation between members of different species. Previous studies in mice and other mammals have focused in particular on evidence for positive or diversifying selection that shapes the evolution of genes that encode sperm-binding proteins expressed in the egg coat or zona pellucida (ZP). By fitting phylogenetic models of codon evolution to data from the 1000 Genomes Project, we identified candidate sites evolving under diversifying selection in the human genes $Z P 3$ and $Z P 2$. We also identified one candidate site under positive selection in $C 4 B P A$, which encodes a repetitive protein similar to the mouse protein ZP3R that is expressed in the sperm head and binds to the ZP at fertilization. Results from several additional analyses that applied population genetic models to the same data were consistent with the hypothesis of selection on those candidate sites leading to coevolution of sperm- and egg-expressed genes. By contrast, we found no candidate sites under selection in a fourth gene (ZP1) that encodes an egg coat structural protein not directly involved in sperm binding. Finally, we found that two of the candidate sites (in C4BPA and ZP2) were correlated with variation in family size and birth rate among Hutterite couples, and those two candidate sites were also in linkage disequilibrium in the same Hutterite study population. All of these lines of evidence are consistent with predictions from a previously proposed hypothesis of balancing selection on epistatic interactions between C4BPA and ZP3 at fertilization that lead to the evolution of coadapted allele pairs. Such patterns also suggest specific molecular traits that may be associated with both natural reproductive variation and clinical infertility. 


\section{Positive selection on human gamete-recognition genes}

2 Michael W. Hart' ${ }^{1}$, Daryn A. Stover ${ }^{2}$, Vanessa Guerra', Sahar V. Mozaffari ${ }^{3}$, Carole Ober ${ }^{3}$, Carina

3 F. Mugal ${ }^{4}$, and Ingemar $\mathrm{Kaj}^{5}$

4 'Department of Biological Sciences, Simon Fraser University, Burnaby, British Columbia,

5 Canada

$6 \quad{ }^{2}$ School of Mathematical and Natural Sciences, Arizona State University Colleges at Lake

7 Havasu City, Lake Havasu City, Arizona, USA

8 32Department of Human Genetics, University of Chicago, Chicago, Illinois, USA

9 'Department of Ecology and Genetics, Uppsala University, Uppsala, Sweden

10 'Department of Mathematics, Uppsala University, Uppsala, Sweden

11 Corresponding author: Michael W. Hart' (mwhart@sfu.ca) 


\section{Abstract}

13 Coevolution of genes that encode interacting proteins expressed on the surfaces of sperm and

14 eggs can lead to variation in reproductive compatibility between mates and reproductive isolation

15 between members of different species. Previous studies in mice and other mammals have

16 focused in particular on evidence for positive or diversifying selection that shapes the evolution

17 of genes that encode sperm-binding proteins expressed in the egg coat or zona pellucida (ZP). By

18 fitting phylogenetic models of codon evolution to data from the 1000 Genomes Project, we

19 identified candidate sites evolving under diversifying selection in the human genes $Z P 3$ and $Z P 2$.

20 We also identified one candidate site under positive selection in $C 4 B P A$, which encodes a

21 repetitive protein similar to the mouse protein ZP3R that is expressed in the sperm head and

22 binds to the ZP at fertilization. Results from several additional analyses that applied population

23 genetic models to the same data were consistent with the hypothesis of selection on those

24 candidate sites leading to coevolution of sperm- and egg-expressed genes. By contrast, we found

25 no candidate sites under selection in a fourth gene $(Z P 1)$ that encodes an egg coat structural

26 protein not directly involved in sperm binding. Finally, we found that two of the candidate sites

27 (in $C 4 B P A$ and $Z P 2$ ) were correlated with variation in family size and birth rate among Hutterite

28 couples, and those two candidate sites were also in linkage disequilibrium in the same Hutterite

29 study population. All of these lines of evidence are consistent with predictions from a previously

30 proposed hypothesis of balancing selection on epistatic interactions between $C 4 B P A$ and $Z P 3$ at

31 fertilization that lead to the evolution of co-adapted allele pairs. Such patterns also suggest

32 specific molecular traits that may be associated with both natural reproductive variation and

33 clinical infertility. 


\section{Introduction}

35 Proteins expressed on the surfaces of gametes mediate several types of cellular interactions at the

36 time of fertilization, including chemoattraction of swimming sperm towards the egg, sperm

37 binding to the egg extracellular coat, lysis or dissolution of the egg coat, and fusion of the

38 gamete plasma membranes. Because those interactions may directly affect reproductive success,

39 the genes encoding such proteins are sensitive to selection and often show evidence of

40 diversifying or positive selection leading to high rates of amino acid substitution (Swanson et al.

41 2001; Swanson and Vacquier 2002; Hamm et al. 2007; Clark et al. 2009; Vicens and Roldan

42 2014). Different adaptive solutions to selection acting on variation in gamete attraction, binding,

43 or fusion can lead to significant population differentiation within species (e.g., Hart et al. 2014),

44 and are implicated in the origin or maintenance of reproductive isolation between species (Coyne

45 and Orr 2004; Palumbi 2009; Lessios 2011; Gavrilets 2014). Understanding the nature of

46 selection on mammalian genes that encode gamete-binding proteins may help to characterize the

47 causes of natural variation in reproductive success, the potential for development of

48 contraceptive vaccines (Lou et al. 1995; Delves, Lund \& Roitt 2002; Meeusen et al. 2007), or the

49 targets of clinical treatment for infertility (Männikkö et al. 2005; Huang et al. 2014).

50 The human genome includes three functional members of the ZP domain gene family

$51(Z P 1, Z P 2$, and $Z P 3)$ that are shared with the mouse genome and encode the peptide portion of

52 three glycoproteins that form the thick, fibrous extracellular coat or zona pellucida surrounding

53 the mammalian egg (Hirohashi et al. 2008; Burnett et al. 2011; Avella, Xiong \& Dean 2013).

54 The mouse genes that encode proteins known to interact with the egg coat (see Turner and

55 Hoekstra 2008a) include Zp3r(also called sp56; Buffone et al. 2008; Wassarman 2009), which is

56 expressed in sperm, localized to the acrosomal vesicle in the sperm head, and binds to the mouse

57 egg coat (Buffone et al. 2008; Wassarman 2009). The mouse Zp3r gene is homologous and

58 syntenic with the human gene encoding the complement component 4 binding protein alpha

59 (C4BPA; OMIM 120830, 1q32.2). In experimental studies, mouse proteins in the sperm head 
60 (including ZP3R) interact with a sperm-binding domain in the C-terminal region of ZP3 (Chen,

61 Litscher \& Wassarman 1998; Williams et al. 2006) and another in the N-terminal region of ZP2

62 (Baibakov et al. 2012; Avella, Xiong \& Dean 2013), but the role and relative significance of

$63 \mathrm{ZP} 3$ versus ZP2 in the specificity of mouse sperm binding is controversial (Litscher, Williams \&

64 Wassarman 2009; Baibakov et al. 2012; Muro et al. 2012; Avella, Xiong \& Dean 2013).

65 Comparative evolutionary studies revealed that $Z p 3$ has evolved under diversifying

66 selection among species in some mammalian clades, and that the signal of positive selection in

67 mouse $Z p 3$ is strongest in the exon that encodes the C-terminal sperm-binding domain (Swanson

68 et al. 2001; Turner and Hoekstra 2006, 2008b). Unfortunately, there is little complementary

69 information about the evolution of $Z p 3 r / s p 56$. In the genomes of rodents, $Z p 3 r$ occurs with

70 C4bpa in a cluster of genes called the regulator of complement activator (RCA) that encode

71 many of the proteins involved in innate immunity (Mayilyan 2012). Evolutionary relationships

72 among genes related to $Z p 3 r$ and C4bpa are complicated by gene duplications, functional

73 divergence, or loss of function leading to pseudogenes (e.g., Rodríguez de Córdoba et al. 1994;

74 de Villena and de Córdoba 1995; Hillarp et al. 1997; Krushkal, Bat \& Gigli 2000). Comparative

75 analyses show strong evidence of diversifying selection acting on many sites in $C 4 B P A$ among

76 humans and other great apes (Cagliani et al. 2016), but the evolution of $Z p 3 r / s p 56$ among mice

77 and other rodents has not been studied (see Morgan et al. 2010, 2017). In general, it has been

78 difficult to determine which sperm-expressed genes mediate the specificity or compatibility of

79 sperm binding to the mammalian egg coat (Turner and Hoekstra 2008a). The role of particular

80 sperm-expressed genes or their epistatic interactions with ZP genes is controversial in mouse

81 studies, which indicate that ZP3R binds to the egg coat but is not required for male fertility

82 (Wassarman 2009; Muro et al. 2012; Claw, George \& Swanson 2014; Wilburn and Swanson

83 2016; Wang et al. 2017). The role of other genes in the RCA cluster related to Zp3r (including

$84 C 4 B P A)$ in fertilization has not been studied directly in humans or other primates.

85 Important evidence for selection acting on epistatic interactions between genes has come

86 from measures of the strength of gametic disequilibrium, or associations between polymorphisms 
87 at physically unlinked loci that encode networks of interacting gene products (Sinervo and

88 Svensson 2002; Single et al. 2007; Qian, Zhou \& Tang 2015). Because such associations are expected to break down at meiosis, their persistence within populations can be ascribed to

90 ongoing selection that favours some functionally advantageous combinations of allele pairs. For

91 genes involved in gamete recognition, such allele pairs may be maintained by a fitness advantage

92 at fertilization (Palumbi 1999; Clark et al. 2009; Buzbas, Joyce \& Rosenberg 2011). Rohlfs,

93 Swanson \& Weir (2010) analyzed nonrandom associations among single nucleotide

94 polymorphisms (SNPs) in 1504 individuals from the 1958 British Birth Cohort study (Wellcome

95 Trust Case Control Consortium 2007). That analysis used two measures of gametic

96 disequilibrium (or linkage disequilibrium, LD) between genotypes at SNPs in the genomic

97 region near human $C 4 B P A$ and near $Z P 3$ (OMIM 182889, 7q11.23). Permutation tests and

98 simulations of selection acting on tag SNPs suggested that some of the pairwise associations

99 between two-locus genotypes were significantly stronger than expected, and might be accounted

100 for by selection favouring particular combinations of $C 4 B P A$ and $Z P 3$ genotypes at fertilization.

101 Rohlfs, Swanson \& Weir (2010) proposed an evolutionary scenario in which sexual conflict

102 between mates over the optimal rate of sperm-egg binding (fast for males, slow for females)

103 leads to cyclical episodes of coevolution between male and female molecular adaptations. In this

104 scenario, selection on male adaptations for enhancing fertilization rates (at $C 4 B P A$ ) is followed

105 by selection on female countermeasures for reducing rates of sperm-egg contact and mitigating

106 the lethal effects of polyspermy on offspring (at ZP3); both parts of this cycle are driven by

107 negative frequency-dependent selection (a form of balancing selection); and the process results

108 in the accumulation of coadapted pairs of $C 4 B P A$ and $Z P 3$ alleles in LD.

109 The scenario and mechanism for human $C 4 B P A-Z P 3$ coevolution proposed by Rohlfs,

110 Swanson \& Weir (2010) can account for polymorphism in both genes and LD between them.

111 That scenario is consistent with other examples of temporal or cyclical variation in selection on

112 genes encoding gamete-binding proteins (Levitan 2012; Wilburn and Swanson 2016), the

113 evidence for widespread balancing selection across the human genome (Andrés et al. 2009), the 
114 proposed role of such balancing selection in the maintenance of $Z p 3$ polymorphisms in mouse 115 populations in nature (Turner and Hoekstra 2008b), and the evidence for ZP3-ZP3R functional 116 interactions at the protein and cellular level during experimental fertilizations of mouse eggs in

117 the lab (Litscher, Williams \& Wassarman 2009). This scenario is also consistent with the general

118 predictions from analogous models of balancing selection acting on cooperating or cheating

119 genotypes in systems of social conflict (similar to systems of sexual conflict), in which negative

120 frequency dependence leads to an evolutionary arms race between adaptations for cooperation or 121 for cheating, maintains polymorphism for both traits, and creates LD between them (Brockhurst 122 et al. 2014; Ostrowski et al. 2015).

123 The specific targets of selection on functional features of sperm-egg binding that could 124 lead to coevolution of $C 4 B P A$ and $Z P 3$ are expected to be nonsynonymous nucleotide 125 polymorphisms that affect amino acid sequence, protein structure, and the functional interaction 126 between proteins at fertilization. However, few of the tag SNPs analyzed by Rohlfs, Swanson \& 127 Weir (2010) were in exons: none of the seven tag SNPs in ZP3 were in coding sequences; two of 128 nine tag SNPs in C4BPA were in coding sequences, but only one of these (rs4844573) was a 129 nonsynonymous SNP encoding a $C 4 B P A$ amino acid difference. As a result, most of these 130 pairwise associations between SNPs may be indirectly implicated in $C 4 B P A-Z P 3$ coevolution, 131 but are probably not the direct targets of selection acting on functional variation between coding 132 sequences expressed on the surfaces of gametes.

133 Here we use data and methods that complement and expand on those of Rohlfs, Swanson $134 \&$ Weir (2010) to revisit the evidence for diversifying selection (and in particular evidence for 135 the effects of balancing selection) on and coevolution among human genes involved in sperm 136 binding to the ZP. Using previously published genome sequences, we focused on coding 137 sequence variation in $C 4 B P A$ and $Z P 3$, and in the $Z P 2$ gene (OMIM $182888,16 \mathrm{p} 12.2)$ that is 138 also implicated in sperm-egg binding (Baibakov et al. 2012; Avella, Xiong \& Dean 2013). As a 139 negative control, we also analyzed $Z P 1$ (OMIM 615774, 11q12.2), which encodes a structural 140 protein that interacts with $\mathrm{ZP} 3$ and $\mathrm{ZP} 2$ to form the fibrous matrix of the zona pellucida but is not 
141 known to interact directly with sperm. We found one candidate site each under episodic

142 diversifying selection in $C 4 B P A, Z P 3$, and $Z P 2$ (but not $Z P 1$ ) in human populations. Other

143 patterns of variation at those three candidate sites were consistent with the hypothesis of

144 balancing selection favouring polymorphisms in those genes. We also found evidence of

145 significant covariation between two of the candidate sites and reproductive success in a

146 previously well-studied human founder population. Altogether the results were consistent with

147 the hypothesis of some form of balancing selection leading to coevolution among these gamete-

148 recognition genes (as proposed by Rohlfs, Swanson \& Weir 2010), and indicate targets of 149 ongoing selection that affect human fertility.

\section{Methods and Results}

\section{Overview}

152 Our motivation for this study came from previous analyses of sperm- and egg-expressed genes

153 with epistatic interactions at fertilization in sea stars, in which we found evidence of selection for

154 amino acid differences between alleles from diverging conspecific populations (Sunday and Hart

155 2013) and from closely-related species (Popovic et al. 2014; Patiño et al. 2016), and strong

156 effects of those amino acid differences on sperm-egg compatibility and fertilization rates in

157 laboratory experiments (Hart et al. 2014). Our goals in this study were similar: to identify

158 specific sites under selection that could account for previously discovered LD between human

$159 C 4 B P A$ and $Z P 3$ (Rohlfs, Swanson \& Weir 2010), extend those analyses to include human $Z P 2$,

160 and identify possible phenotypic effects of those polymorphisms on reproductive success. For

161 those reasons, we used a work flow similar to our previous studies of sea star genes that mediate

162 sperm-egg binding and reproductive success.

163 We first used a phylogenetic model of codon evolution designed to identify individual

164 codons that have experienced episodic or diversifying selection by the estimation of relative rates

165 of nonsynonymous $\left(d_{N}\right)$ and synonymous nucleotide changes $\left(d_{s}\right)$ mapped onto a phylogeny of

166 relationships among sampled haplotypes. The effects of selection are estimated as the ratio of 
167 those relative rates $\left(\omega=d_{N} / d_{s}\right)$ : selection favouring the accumulation of amino acid differences at 168 rates higher than expected from neutral processes (mutation and genetic drift) alone is inferred

169 for codons with $\omega>1$. We carried out this analysis first because it provides a powerful method to

170 identify specific candidate sites under selection, although such codon models were originally

171 developed for comparative analyses of coding sequence divergence between reproductively

172 isolated species (Kryazhimskiy and Plotkin 2008; Mugal, Wolf \& Kaj 2014). These sensitive

173 models of codon evolution can identify small numbers of candidate sites under diversifying

174 selection, and distinguish them from a background of overall purifying selection $(\omega<1)$ or neutral

175 evolution $(\omega \sim 1)$ at other sites (Murrell et al. 2012). In these models, parallel nonsynonymous

176 changes at several times in the evolutionary history of the gene or in several lineages of the gene

177 tree make up much of the signal of high $\omega$ values associated with specific codons under episodic

178 or diversifying positive selection. When applied to conspecific populations, high $\omega$ values might

179 indicate ongoing diversifying (or balancing) selection. Important caveats about that application

180 and interpretation are summarized below.

181 Second, we used population genetic methods to explore variation at candidate sites that 182 might be consistent with the scenario of Rohlfs, Swanson \& Weir (2010) that these genes evolve 183 under selection for sperm-egg compatibility mediated by sexual conflicts of interest. We used 184 several population genetic tests for selection, and we examined patterns of haplotype frequency 185 variation for each gene. These population genetic analyses can be used to corroborate our results 186 from models of codon evolution, and may also help to account for possible false positives that 187 are known to be associated with some models of codon evolution when those models are fitted to 188 data from conspecific populations (Kryazhimskiy and Plotkin 2008; Mugal, Wolf \& Kaj 2014). 189 Third, we searched for covariation between candidate sites under selection and their effect 190 on gamete compatibility. Because sperm-egg binding and fertilization rates are not as readily 191 observed in humans as in sea stars or other organisms with external fertilization, we used proxies 
192 for gamete binding and fertilization success based on family size and birth rate data from a

193 previously well-studied human founder population (Kosova et al. 2012).

194 At several steps in the study our choice of the next analysis to carry out depended on the

195 observations or analytical results from a previous step in the study. This type of data-dependent

196 analysis has been called a "garden of forking paths" (Gelman and Loken 2014) in which the

197 choices of analyses by the researcher (sometimes called "researcher degrees of freedom";

198 Simmons, Nelson \& Simonsohn 2011) are not specified in advance of the study. Researcher

199 degrees of freedom can be used to preferentially report only positive results with significant $P$ -

200 values, and that practice may inflate the apparent strength of results and lead to a high expected

201 rate of false positive discoveries (Forstmeier, Wagenmakers \& Parker 2017). For those reasons,

202 in the sections below we present both the analytical method used and the results of that analysis

203 together for each step in order to show and acknowledge the data-dependent nature of the work

204 flow. Our discoveries should be interpreted as the basis for follow-up studies and analysis, rather

205 than as conclusive tests of the balancing selection and coevolution scenario proposed by Rohlfs,

206 Swanson \& Weir (2010).

207 Phylogenetic models of codon evolution indicate episodic diversifying selection 208 at three candidate sites

209 We obtained 2184 full-length coding sequences for $C 4 B P A, Z P 3, Z P 2$, and $Z P 1$ from 1092

210 individual humans in the Phase 1 release (20110521) of the 1000 Genomes Project database

211 (1000 Genomes Project Consortium, 2012), which includes samples of 14 populations from four

212 continental groups including Asia (Japan, northern and southern China), Europe (Finland,

213 Britain, Spain, Italy, and European Americans from Utah), the Americas (Puerto Rico, Mexico,

214 Colombia), and Africa (Kenya, Nigeria, and African Americans in the southwestern USA).

215 Population sample sizes in Phase 1 were 14-100 individuals (28-200 gene copies). We used the

216 Phase 1 release because these data consist of phased haplotypes suitable for analysis using

217 phylogenetic or coalescent models of codon evolution that employ gene trees. We analyzed the 
218 longer of two transcriptional variants for $Z P 3$. Each alignment was visualized in the Se-Al

219 aligner (Rambaut 2002), trimmed to coding sequence only, and exported for downstream 220 analysis.

221 We used blastn searches of the GenBank nonredundant nucleotide database, and the 222 SMART tool (Letunic et al. 2015) for characterizing protein sequence domains, to confirm the 223 identity of each gene downloaded from the 1000 Genomes database; we used the UCSC genome 224 browser (Kent et al. 2002) to confirm the synteny of each gene with the corresponding mouse 225 reference genes. The C4BPA protein consists of eight complement control peptide domains 226 (CCP, also known as sushi domains; reviewed by Ermert and Blom 2016) each approximately 227 55-60 amino acids in length. The ZP genes each encode several distinctive protein domain types, 228 including a short signal peptide, a ZP domain about 260 amino acids in length, a furin-type 229 peptide cleavage sequence, and a propeptide following the cleavage motif that includes a 230 transmembrane domain involved in cytoplasmic trafficking of the protein into the extracellular 231 egg coat (reviewed by Monné and Jovine 2011; Wilburn and Swanson 2016).

232 We used the branch-site model of codon evolution implemented in MEME (mixed-effects 233 model of evolution; Murrell et al.2012) to identify sites in each gene with high relative rates of 234 nonsynonymous change that might evolve under diversifying selection. We used tools on the

235 Datamonkey web interface (Delport et al. 2010) to screen each sequence alignment for 236 recombination and to choose a model of nucleotide evolution (Kosakovsky Pond et al. 2006). We 237 then estimated a phylogeny using the maximum-likelihood method (and the best-fit nucleotide 238 model) in MEGA 5 (Tamura et al.2011), and used that gene tree in a MEME analysis. The 239 MEME method is similar to the branch-sites models in the PAML software package (Yang 240 2007), but has several advantages including the ability to model variation among codons in both 241 the synonymous and nonsynonymous rates of change, and greater sensitivity to identify targets 242 of selection $(\omega>1)$ at a small number of functionally important codons among only some lineages 243 in a gene tree. 
The MEME analyses identified 1-3 codons in each of the three genes known to be involved in sperm-egg binding $(C 4 B P A, Z P 3, Z P 2)$ that showed evidence of episodic diversifying selection (positive selection on those individual codons among some lineages in the gene tree; Table 1). For each of those codons, a likelihood ratio test indicated a significantly better model fit $(P<0.05)$ when the nonsynonymous rate $\beta$ at those sites was allowed to exceed the synonymous rate $\alpha$ in comparison to a null model with the constraint $\beta=\alpha$.

In $C 4 B P A$ there was one candidate site under selection (C4BPA 300; rs4844573) in the Nterminal region of the fifth sushi domain (Table 1) that showed a nonsynonymous polymorphism

252 (a second-position transition encoding a threonine-isoleucine polymorphism; Table 2). Notably, 253 this was the only nonsynonymous site included among the tag SNPs analyzed by Rohlfs,

254 Swanson \& Weir (2010), and was also one of the sites found by Rohlfs, Swanson \& Weir (2010) to be in $\mathrm{LD}$ with a polymorphism near $Z P 3$.

There were three sites under selection in ZP3 (Table 1), including one near the signal sequence $(Z P 331$; rs2286428) in a protein domain of unknown function that is not part of the modeled protein structure based on x-ray crystallography of chicken ZP3 (Han et al. 2010;

259 Monné and Jovine 2011), and a second site in the C-terminal propeptide region (ZP3 371;

260 rs200481427) downstream of the peptide cleavage site and thus not part of the mature functional

261 protein in the egg coat. Because these two sites are in protein domains not expected to be

262 involved in sperm binding, we focused our subsequent analyses of $Z P 3$ on a third candidate site 263 (ZP3 315; rs2906999) that showed a nonsynonymous polymorphism (a first-position transition 264 encoding a proline-serine polymorphism) in the known sperm-binding domain (Table 2). In the 265 structural model of chicken ZP3 by Han et al. (2010), this candidate site is in a C-terminal region 266 of secondary structure that is strongly constrained by three disulphide bridges among six highly 267 conserved cysteine residues upstream $\left(\mathrm{C}_{6}, \mathrm{C}_{8}\right)$ and downstream $\left(\mathrm{C}_{9}-\mathrm{C}_{12}\right)$ of human $Z P 3315$ (see 268 Monné and Jovine 2011).

269 There was one candidate site under selection in $Z P 2$ in the $\mathrm{N}$-terminal part of the predicted 270 protein (ZP2 36; rs2075520) that showed a nonsynonymous polymorphism (a second-position 
271 transversion encoding a valine-glycine polymorphism; Table 2). Like the candidate site in $Z P 3$

272 noted above, this single codon under selection in ZP2 was also in the known sperm-binding

273 domain.

274 In contrast to those results for three genes that are expected to be involved in sperm-egg

275 binding, we found no candidate sites under selection in MEME analyses of $Z P 1$ variation. All

276 likelihood ratio tests suggested that the nonsynonymous rate $\beta$ was not significantly different

277 from the synonymous rate at any individual codon (all $P>0.10$ ). That result suggests that

278 diversifying selection acting on ZP genes may be limited to those genes $(Z P 3, Z P 2)$ known to be

279 involved in sperm binding. For those reasons we focused the remainder of our analyses on the

280 three candidate sites under selection in $C 4 B P A, Z P 3$, and $Z P 2$.

281 Population genetic analyses also indicate selection at the same candidate sites

282 Models of codon evolution like MEME were originally developed to analyze sequences from

283 highly divergent lineages such as reproductively isolated species that have diverged on a long

284 time scale and for which phylogenetic relationships can be characterized accurately (Goldman

285 and Yang 1994; Muse and Gaut 1994). An important model assumption is that differences

286 between those sampled lineages are fixed substitutions (possibly caused by selection) and not

287 segregating polymorphisms (influenced by genetic drift). False-positive results can arise when

288 the model parameters are estimated from alignments of closely-related conspecific haplotypes

289 that have diverged on short time scales: if the data include segregating nonsynonymous

290 polymorphisms that arose as recent mutations under weak purifying selection with insufficient

291 time for selection to eliminate those mutations, then a signal of high $\omega$ may be falsely ascribed to

292 selection favouring the accumulation of nonsynonymous differences at some codons or in some

293 lineages. Similarly, models of codon evolution (like MEME) based on phylogenies may be

294 misled by uncertainty about relationships among closely-related haplotypes sampled within a

295 single species, and an important source of such uncertainty is recombination. These potential

296 problems of false discovery in codon-model analyses of population data are well known (Stajich 
297 and Hahn 2005; Hofer et al. 2009; Kryazhimskiy and Plotkin 2008; Anisimova and Liberles

298 2012; Mugal, Wolf \& Kaj 2014). The high $q$ values in Table 2 ( $q=1$ for all five sites with high $\omega$

299 values identified by the MEME method) are also consistent with the expected high false-

300 discovery rate, and suggest that our MEME results could include some false positives.

301 We used three approaches to test the reliability of our MEME results. First, we used an 302 additional model of codon evolution called omegaMap v.0.5 (Wilson and McVean 2006) that

303 bridges the gap between interspecific analyses of selection based on phylogenies and

304 intraspecific analyses of selection based on population samples. The omegaMap model is fitted

305 to data by estimating mutation rate and recombination rate parameters as well as $\omega$ for different

306 regions of a gene alignment using a sliding window method. Rather than mapping those rates

307 onto a single phylogenetic tree, omegaMap uses a population genetic approximation of the

308 coalescent within a Bayesian framework that accounts for uncertainty about genealogical

309 relationships among haplotypes.

310 The omegaMap algorithm is computationally intensive and would not converge on a model

311 fit using the full alignment for any of the three genes we analyzed. Instead we analyzed a

312 reduced dataset for each gene consisting of the two haplotypes for one individual selected at

313 random from each population in the 1000 Genomes Phase 1 release. All omegaMap results

314 reported were based on this reduced dataset of 28 haplotypes total. We used inverse or improper

315 inverse priors for model parameters, and allowed both the selection and recombination

316 parameters to vary along the alignment. We carried out a series of preliminary analyses to find

317 suitable prior ranges, parameter starting values, burn-in lengths, and block widths for the sliding-

318 window analyses. We then ran the final MCMC model fit for 500,000 or 700,000 iterations, and

319 sampled the model parameter values at intervals of 500 or 700 steps.

320 In comparison to MEME model parameter values (Table 2), omegaMap identified the same

321 candidate sites under diversifying selection, but gave much lower (and more realistic) estimates

322 of $\omega(2-4$; Fig. 1$)$. The posterior probabilities of positive selection $(\omega>1)$ at the three candidate 
323 sites were high ( $P>0.8$; Fig. 1). Those results suggest that our MEME analyses correctly

324 identified candidate sites under selection, but may have overestimated the magnitude of the 325 response to selection.

326 Second, we used the 1000 Genomes Selection Browser v.1.0 (http://hsb.upf.edu; Pybus et 327 al.2014) to carry out population genetic tests for selection. This tool implements a series of 328 selection analyses applied to population samples from Caucasian Europeans in Utah (CEU), Han 329 Chinese from Beijing (CHB), and Yorubans from Ibidan, Nigeria (YRI), that are representative 330 of continental population groups in the 1000 Genomes database. Among the methods available 331 using this tool, we chose two based on population allele frequency differences including pairwise $332 \quad F_{\mathrm{sr}}$ between populations and global $F_{\mathrm{sr}}$ among all three populations (Weir and Cockerham 1984);

333 two based on linkage disequilibrium among sites within each gene including the integrated 334 haplotype score within each population sample (iHS; Voight et al. 2006), and extended 335 haplotype homozygosity in comparisons between pairs of populations (XP-EHH; Sabeti et al. 336 2007; see also Zhong et al. 2011); and three based on the allele frequency spectrum within each 337 population including Tajima's $D$ (Tajima 1989), the composite likelihood ratio test (CLR; 338 Nielsen et al. 2005), and Fay and Wu's $H$ (Fay and Wu 2000). Although $F_{\mathrm{s \tau}}$ values for a single 339 locus may be interpreted as measures of population differentiation when compared to a neutral 340 expectation (based on permutation), such values can also be interpreted as indices of selection 341 when compared to other loci across the genome (sometimes called outlier tests; Foll and 342 Gaggiotti 2008; Holsinger and Weir 2009). All seven tests are designed to detect significant 343 departures from expectations based on the effects of neutral processes alone (mutation, genetic 344 drift, recombination; reviewed by Vitti, Grossman \& Sabeti 2013). They are typically interpreted 345 as tests of directional or purifying selection leading to differences among populations, but the 346 tests may also be sensitive to the effects of diversifying or balancing selection when the results of 347 such selection also resemble an incomplete selective sweep. We analyzed a $2 \mathrm{Mbp}$ interval 348 around each candidate site under selection in order to characterize the evidence for selection 349 associated with all variable sites. The 1000 Genomes Selection Browser estimates the $P$-value 
350 associated with each test statistic for each candidate site under selection as its rank score relative

351 to other sites in the $2 \mathrm{Mbp}$ interval (the proportion of all other sites with a test statistic value as

352 high or higher than the candidate site). Because that database uses the GRCh37/hg19 reference

353 genome, we centered each $2 \mathrm{Mbp}$ interval around the coordinates for each of the candidate sites

354 under selection in that reference, including chr1:207,304,900 (rs4844573, C4BPA),

355 chr7:75069811-77069811 (rs2906999, ZP3), and chr16:21,222,672 (rs2075520, ZP2), instead of

356 the coordinates shown in Table 2 for each of those sites (in the GRCh38/hg38 reference

357 genome).

358 We found evidence for selection acting on those three candidate sites from two of the three

359 types of tests. Four of the tests based on population differentiation (three of six pairwise $F_{\mathrm{sT}}$

360 values, and one of two global $F_{\mathrm{sT}}$ values) suggested significant population allele frequency

361 differences at both the candidate site in $C 4 B P A$ and the candidate site in $Z P 2$ (Table 3). All three

362 of the significant results for pairwise $F_{\mathrm{sT}}$ tests involved comparisons between the African sample

363 (YRI) and one of the non-African samples (for ZP2) or both of the non-African samples (for

$364 C 4 B P A$ ), and for $C 4 B P A$ we found significant global differentiation as well. Overall evidence

365 for selection was stronger for the candidate site in $C 4 B P A$ (three of four significant test results)

366 than for the candidate site in $Z P 2$ (one of four).

367 We also found evidence of selection in some tests based on measures of LD across sites

368 within the $C 4 B P A$ and $Z P 2$ genes. Like the tests based on population differentiation, evidence

369 for selection was limited to variation within the African sample (iHS) and comparisons between

370 the African and non-African samples (XP-EHH) (Table 3), and was stronger for the candidate

371 site in $C 4 B P A$ (three of six significant test results) than for the candidate site in $Z P 2$ (no

372 significant test results). This evidence for selection acting on $C 4 B P A$ variation seems convincing

373 because the two tests differ in their sensitivity to detect homozygosity and LD caused by

374 selection acting in the recent (iHS) or the more distant past (XP-EHH) (Vitti, Grossman \& Sabeti

375 2013), but both tests indicated significantly greater extent of homozygosity around rs4844573 in

$376 C 4 B P A$ compared to expected extent of homozygosity under neutral processes alone. 
By contrast, none of the individual tests based on properties of the allele frequency

spectrum (Table 3) suggested significant departures from neutral evolution for the candidate sites

in $C 4 B P A$ or $Z P 2$. Values for all three test statistics were small within each of the three

380 population samples, and none were unusual (all $P>0.09$ ) relative to test statistic values for other variable sites in the $2 \mathrm{Mbp}$ interval surrounding both of the candidate sites.

variable site in the 1000 Genomes Selection Browser database, so we used other methods to

384 implement some of the same tests for that missing candidate site. We focused on the two classes of tests (based on population differentiation, and based on LD) that did indicate some evidence of selection on the other two candidate sites in tests using the 1000 Genomes Selection Browser.

387 We calculated pairwise $F_{\mathrm{sT}}$ between the CEU, CHB, and YRI populations (all $\left.F_{\mathrm{sr}}<0.040\right)$ and 388 global $F_{\mathrm{sr}}$ among all three populations $\left(F_{\mathrm{sr}}=0.021\right)$ for the candidate site in $Z P 3$ using the method of Raymond and Rousset (1995). When compared to the ranked scores for $F_{\mathrm{sT}}$ values at other sites in the $2 \mathrm{Mbp}$ interval spanning that candidate site (rs2906999), the global $(P=0.53)$ and all pairwise $F_{\mathrm{s \tau}}$ comparisons (all $P>0.38$ ) were not significant. Those comparisons do not indicate selection on the candidate site in $Z P 3$ leading to population allele frequency differences. and XP-EHH values for the candidate site in $Z P 3$. Unlike the 1000 Genomes Selection Browser, 395 selscan calculates an unstandardized test statistic value (for iHS, or XP-EHH) and then 396 characterizes the significance of that value by binning together SNPs with similar allele 397 frequencies (we used 20 bins), and calculating a normalized test statistic value (a $z$-score) 398 relative to normally-distributed test statistic values for all other SNPs in the same bin with a 399 mean of zero and standard deviation of one. Among those six tests (three iHS values for 400 individual populations, three pairwise XP-EHH values), only one was significant: the iHS score 401 for the $\mathrm{CHB}$ population sample (iHS=2.423, $z=2.353, P=0.018$ ). Other iHS scores for the 402 candidate site in $Z P 3$ were lower (iHS $<2.05, P>0.05)$, and all XP-EHH scores were low $(<0.6)$ 403 and not significant $(P>0.6)$. Like the $F_{\mathrm{sT}}$ tests, the analyses based on LD provide only limited 
404 evidence of selection on the candidate site in ZP3 leading to extensive homozygosity around that 405 site.

Third, we used McDonald-Kreitman tests (McDonald and Kreitman 1991) to assess the 407 relative frequency of non-synonymous polymorphisms within human populations, and compare 408 them to the relative frequency of non-synonymous fixed substitution differences between 409 humans and chimpanzees. These tests are typically interpreted as evidence of positive selection 410 favouring functional (nonsynymous) divergence between species when the tests reveal a high 411 relative rate of interspecific nonsynonymous substitutions. However, the same test can also be 412 used to identify evidence of balancing selection favouring the maintenance of functional 413 (nonsynonymous) diversity within species in the form of a high relative rate of intraspecific 414 nonsynonymous polymorphisms (e.g., Schoville, Flowers \& Burton 2012). The test is 415 conservative because it compiles evidence across all sites into a single test statistic for each gene, 416 so a significant result would be strong evidence for balancing selection, but nonsignificant results 417 might be ascribed to weak sensitivity to detect the effects of selection on one candidate site in 418 each gene. We used a single chimpanzee (Pan troglodytes) haplotype from GenBank for each gene (XM_514156.4,XM_016929619.1,XM_009453354.2); the chimpanzee and human coding 420 sequences were the same length for each gene, so no additional alignment was required. We used 421 the method of Egea, Casillas \& Barbadilla (2008) to calculate the test statistic and associated $P$ 422 values. For all three genes, a large proportion (63-73\%) of human polymorphisms was 423 nonsynonymous (Table S1). However, in all three tests the proportion of nonsynonymous 424 polymorphisms was not significantly different from the proportion of nonsynonymous fixed 425 differences in the $2 \times 2$ contingency table (all $P>0.18$ ), thus none of the tests gave evidence of 426 balancing selection.

427 Unlike Rohlfs, Swanson \& Weir (2010), we did not use measures of LD based on allelic or 428 genotypic associations between pairs of candidate sites themselves in the 1000 Genomes Project 429 data to assess the strength of selection acting on those three genes because such tests were 430 expected to be weak in this case. Brown (1975) showed that, for simple allelic associations, the 
431 power to reject the null hypothesis of linkage equilibrium was low for population sample sizes

432 like those in Phase 1 of the 1000 Genomes Project (all samples $\leq 100$ ), unless the true underlying

433 LD was very strong. Similarly, Rohlfs, Swanson \& Weir (2010) showed that the power to detect

434 genotypic associations was also low for small samples $(<200)$ unless the value of the selection

435 coefficient for genotypic associations was high ( $>>0.4)$. We avoided the creation of larger sample

436 sizes by pooling population samples from the 1000 Genomes Project data because such pooled

437 samples may show LD among unlinked sites as an artifact of population differentiation rather

438 than as a consequence of selection for allele or genotype pairs. For those reasons, we were not

439 able to compare directly the patterns of LD among three candidate sites under selection in the

440 (small) 1000 Genomes Project samples to the previously documented patterns of LD between

441 two of those candidate sites in the (larger) 1958 British Birth Cohort sample (Rohlfs, Swanson \&

442 Weir 2010).

443 All three candidate sites under selection are balanced polymorphisms

444 We used the parsimony network method in TCS (Clement, Posada \& Crandall 2000) to illustrate

445 both the frequencies and phylogenetic relationships among all 2184 gene copies for each gene.

446 Each of those three networks included many closed circuits that indicated uncertainty about

447 phylogenetic relationships among closely related gene copies. To illustrate the patterns more

448 clearly, we pruned each of the closed circuits (and reduced the network for each gene down to

449 one example genealogy) by favouring connections to more common haplotypes (and pruning

450 edges leading to less common haplotypes).

451 We found similar patterns of frequency variation and phylogenetic relationships among

452 haplotypes across all three genes (Fig. 2). In both $C 4 B P A$ and $Z P 3$ (Fig. 2A, B), there were two

453 most common haplotypes. The two C4BPA common haplotypes differed only by the

454 nonsynonymous difference at the candidate site in the fifth sushi domain (C4BPA 300); they

455 accounted for $36.7 \%$ and $35.4 \%$ of 2184 gene copies, compared to the much lower frequencies of

45645 other haplotypes. Similarly, the two $Z P 3$ common haplotypes differed from each other only 
457 by the nonsynonymous difference at the candidate site in the ZP3 sperm-binding domain ( $Z P 3$

458315 ), and accounted for $35.9 \%$ and $26.6 \%$ of all chromosomes, compared to lower frequencies of 45963 other haplotypes.

460 We found a slightly more complex pattern of variation among $Z P 2$ haplotypes (Fig. 2C), 461 including four common haplotypes plus 40 less common haplotypes. A single nonsynonymous 462 difference at the candidate site in the ZP2 sperm-binding domain (ZP2 36) distinguished the two 463 pairs of common haplotypes from each other. The two common haplotypes distinguished by the 464 candidate site under selection accounted for $14.0 \%$ and $26.1 \%$ of all chromosomes; each of those 465 haplotypes differed from another common haplotype by a single synonymous change (at a 466 different codon). The four common haplotypes together accounted for $92.3 \%$ of chromosomes. 467 These patterns of diversity for each gene, characterized by a pair of common haplotypes that 468 differ only by a single nonsynonymous difference at a candidate site under selection, are 469 consistent with the predictions of the balancing selection hypothesis of Rohlfs, Swanson \& Weir 470 (2010).

471 In order to infer the direction of nucleotide changes at each candidate site in those 472 phylogenetic networks, we used comparisons to other great ape reference genomes (chimpanzee, 473 gorilla, orangutan) in the UCSC genome browser (Kent et al. 2002). We found that for each 474 candidate site the chimpanzee, gorilla, and orangutan reference genomes as well as the mRNA 475 sequences from bonobos all had the same single human allele (C4BPA 300 Thr; ZP3 315 Pro; $476 Z P 236 \mathrm{Val})$, which suggested that the alternate allele found in humans (C4BPA 300 Ile; ZP3 477315 Ser; ZP2 36 Gly) is derived (Table 2). Mapping those ancestral and derived states onto the 478 gene genealogies allowed us to infer the directions of changes (Fig. 2). In two cases (C4BPA, $479 Z P 2)$, the minor allele summed over all populations was also the ancestral allele; in the third case 480 (ZP3), the ancestral and derived allele frequencies were nearly identical and the minor allele (by 481 a small difference) was the derived allele (Table 4). All three genes showed multiple changes at 482 the candidate site. On the gene trees shown in Fig. 2 these included two cases of parallel changes 483 from the ancestral allele (C4BPA $300 \mathrm{Thr}$; ZP3 315 Pro) to the derived allele (C4BPA 300 Ile; 
$484 \quad Z P 3315$ Ser), and one case of a secondary reversal from the derived allele (ZP2 36 Gly) back to 485 the ancestral allele (ZP2 $36 \mathrm{Val})$. Such parallelisms may account for the signal of selection found 486 in our analyses using models of codon evolution (MEME, omegaMap) that are sensitive to the 487 effects of multiple episodes of selection on the same codon (e.g., Turner and Hoekstra 2006).

488 Vahdati and Wagner (2016) argued that such parallelisms are a reliable signal of positive 489 selection (but possibly not balancing selection) in genome scans of protein-coding sequences.

490 The discovery of similar patterns of parallelism among $C 4 B P A, Z P 3$, and $Z P 2$ is also consistent 491 with the hypothesis of selection acting on epistatic interactions among these three genes.

492 Balanced polymorphisms at candidate sites have unusually high minor allele 493 frequencies

494 High frequencies for both ancestral and derived alleles, and minor allele frequency (MAF) 495 differences between African and non-African populations, may arise by neutral demographic 496 processes instead of (or in addition to) the effects of selection (Hofer et al. 2009; Coop et al. 497 2009). To explore that possibility, we carried out two tests.

498 First, we compared the observed MAFs at the three candidate sites under selection to the 499 expected occurrence of sites with such high MAFs under a purely neutral model. This is a 500 relatively stringent test given the expectation that nonsynonymous mutations in coding 501 sequences are less likely than other mutations to evolve under neutrality. For each candidate site 502 under selection we compared the observed range of MAFs in African versus non-African 503 population groups (Table 4) to a neutral expectation based on a Poisson random field framework 504 (Kaj and Mugal 2016). We used the minimum MAFs for each gene from the four continental 505 population groups (Table 4$)$, and those MAFs $(0.185,0.411,0.273)$ serve as a lower bound and 506 thus make for a more conservative test.

507 We computed for each gene the expected number of sites at which the derived allele exists 508 in both populations with an intermediate frequency in the range $[\mathrm{x}, 1-\mathrm{x}]$. We let $f_{\mathrm{x}}$ be defined by 
$509 f_{\mathrm{x}}(\mathrm{y})=1$, if $\mathrm{x}<\mathrm{y}<1-\mathrm{x}$, and $f_{\mathrm{x}}(\mathrm{y})=0$ otherwise. Then $f_{\mathrm{x}} \in F_{0}$ (see Kaj and Mugal 2016), and the

510 expected number of such sites has the form $\theta H_{x}$, where

$$
H_{x}=\int_{0}^{1} \mathbb{P}_{y}^{0}\left(x<\xi_{t}<1-x\right)^{2}\left(\frac{2}{y}\right) d y
$$

512 The function $\mathbb{P}_{y}^{0}\left(x<\xi_{t}<1-x\right)^{2}$ is known (in terms of hypergeometric sums) so we can find

$513 H_{x}$ numerically. We computed $H_{x}$ for each gene with demographic conditions characteristic of the

514 scenario for the late-Pleistocene migration of early modern humans out of Africa (population

515 size $N=5000$; population divergence time $t=0.5$ coalescent units). Values of $H_{x}$ were 0.8761 ,

5160.0699 , and 0.2149 for $C 4 B P A, Z P 3$, and $Z P 2$, respectively. We then used the numerical solution

517 for $H_{x}$ to calculate the probability $p(x)=1-\exp \left(-\theta H_{x}\right)$ of observing at least one site that has

518 intermediate allele frequencies in $[\mathrm{x}, 1-\mathrm{x}]$ under selectively neutral conditions for each of the

519 three candidate sites (Table 4). For those calculations we used values of $\theta$, the population

520 mutation rate per gene, based on the 1092 individuals of the 1000 Genomes phase 1 data

$521(1.6333,1.5727$, and 1.9962 for $C 4 B P A, Z P 3$, and $Z P 2$, respectively). This approach leads to

522 values for $p(x)$ that are all relatively high $(0.7609,0.1042,0.3488$, respectively). Those high

523 probabilities suggest that the observed MAFs for those three candidate sites in the African

524 population group might have arisen by neutral processes alone under those demographic model

525 conditions (the out-of-Africa migration of early modern humans).

526 Second, we compared MAFs at the three candidate sites under selection (summed over all

527 populations) to MAFs at other nonsynonymous SNPs on the same three human chromosomes. In

528 contrast to the analyses described above, this may be a less stringent (and more realistic) test

529 given the expectation that most nonsynonymous mutations in coding sequences are likely to

530 evolve under purifying selection. In order to standardize that comparison across candidate sites

531 on three different chromosomes, we examined a window of $90.3 \mathrm{Mbp}$ (the length of chromosome

53216 , the shortest of the three chromosomes studied here) spanning each of the candidate sites, and

533 downloaded MAFs for all biallelic nonsynonymous SNPs in the 1000 Genomes Phase 1 release 
534 (3593 SNPs total, including the three candidate sites). We characterized the significance of those

535 balanced polymorphisms by estimating the proportion of other nonsynonymous SNPs on each

536 chromosome that had MAFs greater than the MAF observed for the candidate site on that

537 chromosome.

538 The high MAFs at each candidate site were unusual compared to all other nonsynonymous

539 SNPs in a 90.3 Mbp window spanning each of those candidate sites. In all three cases, the

540 frequency of the minor allele at the candidate site was higher than the MAFs at more than $95 \%$

541 of all other nonsynonymous SNPs on the same chromosome (Table 4). In the case of the ZP3

542 candidate site, no other nonsynonymous SNP in the same region of chromosome 7 showed such

543 a high MAF. For each candidate site, the ancestral allele frequency tended to be higher in

544 African samples compared to all non-African samples (Table 4), but both alleles at each

545 candidate site were found in all populations. These unusual balanced polymorphisms are

546 consistent with the predicted effects of episodic or diversifying selection that favours

547 functionally divergent alleles at each candidate site, and maintains each allele at high frequency

548 within populations. However, the differences between African and non-African population

549 samples at all three candidate sites are also consistent with other hypotheses based on selection

550 (such as an incomplete selective sweep in some or all populations).

551 The two tests together suggest that we cannot entirely reject neutrality as the cause of high 552 observed MAFs at the three candidate sites (based on a stringent comparison to neutrality using

553 calculations in the Poisson random field framework), but we find evidence for either relaxed

554 selective constraints or diversifying selection (based on a more realistic comparison to expected

555 variation under purifying selection at other nonsynonymous sites). Those results indicate that we

556 should be cautious in interpreting the causes of allele frequency variation at those three candidate

557 sites, but they do not contradict other evidence for diversifying selection acting on those three

558 candidate sites.

559 Variation in fertility is associated with candidate sites under selection 
560 To test for covariation between polymorphisms at candidate sites and measures of fertility we 561 assessed genotype-phenotype associations in a founder population, the Hutterites (see Kosova, 562 Abney \& Ober 2010; Kosova et al. 2010; Kosova et al. 2012), in which both reproductive data 563 and genome-wide genotype data were available. As in previous analyses (e.g., Kosova et al. 564 2010), we focused on two measures of fertility: family size (number of births) in 361 families; 565 and birth rate (calculated as [number of births - 1]/[sum of all interbirth intervals]) in 341 566 families with at least two children. We analyzed associations between these fertility measures 567 and two of the three candidate sites (in $C 4 B P A$ and $Z P 2$ ); genotypes for the third candidate site 568 (in ZP3) were not available for most individuals in the sample because that specific SNP 569 (rs2906999) was not included in the SNP chip used to screen genetic polymorphisms in the 570 original study (Kosova et al. 2012). We estimated genotype-phenotype associations using a 571 linear mixed model as implemented in GEMMA (Zhou and Stephens 2014). This model allowed 572 us to account for the known pedigree relatedness among individuals in the sample. Following 573 Kosova et al. (2012), we included wife's birth year, her age at marriage, and the time from 574 marriage to the most recent birth as covariates in the linear models because these covariates 575 make substantial non-genetic contributions to fertility variation among Hutterite men and 576 women. For each family we characterized the wife's genotype at the $Z P 2$ candidate site and the 577 husband's genotype at the C4BPA candidate site as having zero, one, or two derived alleles. We 578 carried out a series of four parallel analyses in which we first asked whether variation in the 579 wife's $Z P 2$ genotype or the husband's $C 4 B P A$ genotype was significantly associated with 580 residual variation in family size or birth rate. We then repeated those analyses by including the 581 spouse's genotype at the other candidate site as an additional covariate, and by adding an 582 interaction term for covariation between the two individual genotypes.

583 Although these post hoc analyses of functional variation were carried out after the 584 identification of candidate sites under selection in $C 4 B P A$ and $Z P 2$, and the original genetic 585 screens were not carried out with these genetic variants in mind, the analyses provide an 586 important check on our inferences based on phylogenetic and population genetic methods. The 
587 models of codon evolution that we used to identify candidate sites under selection are only able

588 to infer the effects of selection that acted in the past, and may have a high risk of false

589 discoveries. One powerful source of evidence for ongoing (rather than past) balancing selection

590 on such sites is their association with phenotypic variation related to fitness in present-day

591 populations (Key et al. 2014). Such tests also can directly address the possibility that candidate

592 sites under selection are false positives. Association studies in groups such as the Hutterites can

593 be especially useful because fertility is generally high among Hutterite couples, and the

594 Hutterites' communal lifestyle and shared religious and cultural practices minimize variation in 595 other non-genetic contributions to fertility (e.g., Kosova et al. 2010).

596 We found a significant association between the wife's $Z P 236$ genotype and variation in

597 family size ( $P=0.040)$ (Fig. 3A). Adding the husband's $C 4 B P A 300$ genotype as a covariate, with

598 or without an interaction term between the two genetic variables, did not improve the model fit 599 and reduced the $P$ value associated with the wife's $Z P 236$ genotype. There was no effect of the 600 wife's ZP2 36 genotype on birth rate, with or without an interaction term between the partners' 601 genotypes.

602 By contrast, there was a significant effect of the husband's C4BPA 300 genotype on 603 variation in birth rate $(P=0.020 ;$ Fig. $3 B)$. Similar to the analyses described above, adding the 604 wife's $Z P 236$ genotype as a covariate did not improve the model fit. The husband's $C 4 B P A 300$ 605 genotype had no significant association with variation in family size.

606 Genotypes at these two candidate sites influenced fertility in opposite directions and were 607 associated with different components of fertility. For the C4BPA candidate site, a larger number 608 of derived male $C 4 B P A 300$ Ile alleles was associated with increased fertility (higher birth rate, 609 or shorter interbirth intervals); but for the $Z P 2$ candidate site, a smaller number of derived female 610 ZP2 36 Gly alleles was associated with increased fertility (larger family size, or more births).

611 Although these two candidate sites did not appear to have interactive effects on either family size 612 or birth rate, linkage disequilibrium between them would be consistent with the expectation that 613 selection acts on the functional interaction between C4BPA and ZP2 at fertilization. Using the 
614 same measure of genotype associations (the contingency table method) used by Rohlfs, Swanson

$615 \&$ Weir (2010), we found significant LD between those two candidate sites in this large sample

616 of Hutterite families $\left(\chi^{2}=13.72, P=0.0082\right)$. This finding reflected both the low observed

617 frequency of double homozygotes for the two ancestral alleles and the high observed frequency

618 of double homozygotes for the derived C4BPA 300 allele (Ile/Ile) with the ancestral $Z P 236$

619 allele (Val/Val) (Table 5). Overall, the evidence for LD between these two candidate sites and

620 their individual effects on reproductive success are consistent with both our phylogenetic and

621 population genetic analyses, and with the hypothesis that these genes evolve under some form of

622 balancing selection that favours divergent alleles at specific functionally important sites under

623 selection at fertilization.

624 Discussion

625 Selection on human gamete traits

626 Genes underlying morphological, behavioural, physiological, and molecular traits that affect

627 human mate choice and reproductive success are expected to evolve in response to sexual

628 selection or sexual conflict over the specificity or choosiness of mating (Andersson and

629 Simmons 2006; Dixson 2009), and those traits could include molecules expressed on the surfaces

630 of sperm and eggs (Swanson and Vacquier 2002; Palumbi 2009). Behavioural and morphological

631 traits involving premating interactions are widely viewed (e.g., Puts 2010) as the more important

632 targets of sexual selection on human mate choice compared to molecular traits involving

633 interactions between gametes after mating. However, molecular evidence for selection on genes

634 encoding human sperm-egg binding molecules and other reproductive proteins (Swanson et al.

635 2001; Swanson and Vacquier 2002; Hamm et al. 2007; Meslin et al. 2012; Good et al. 2013)

636 indicates that human populations also respond to selection on reproductive success at the level of

637 biochemical and cellular interactions within the female reproductive tract at the time of

638 fertilization (Vacquier, Swanson \& Lee 1997; Swanson and Vacquier 2002). 
639 Genetic and phenotypic evidence for coevolution under selection

640 An important area of focus for studies of the human molecular mating system has been zona

641 pellucida glycoproteins on the egg and ZP-binding proteins in sperm (Litscher, Williams \&

642 Wassarman 2009; Wassarman 2009; Avella, Xiong \& Dean 2013). Using data and methods that

643 complement the population genetic analyses of this system by Rohlfs, Swanson \& Weir (2010),

644 we found patterns that are consistent with (1) their hypothesis that LD between two physically

645 unlinked gamete-recognition genes is caused by balancing selection favouring alternative pairs

646 of alleles, and (2) the functional interpretation that such selection may arise from allelic

647 differences at each of those interacting genes through their influence on gamete binding and

648 fertilization success.

649 The significant functional correlations between maternal or paternal genotypes at candidate

650 sites and variation in both family size and birth rate (Fig. 3) suggest that these candidate sites are

651 indeed targets of selection and are not false positives. In the available data, allelic effects at these

652 two candidate sites in $C 4 B P A$ and $Z P 2$ appear to be additive, similar to the additive effects of

653 SNP markers on fertility identified through genetic association analyses of Hutterite families

654 (Kosova et al. 2012). Moreover, one of those candidate sites (C4BPA 300) was also discovered

655 as a tag SNP associated with $Z P 3$ variation using a different method (LD calculations) in a

656 different study population (Rohlfs, Swanson \& Weir 2010). The overall consistency in the

657 identification of sites under selection, their associations with reproductive variation from three

658 different methodological approaches (genotypic association, models of codon evolution, LD), the

659 discovery of the same candidate sites using two different codon-model methods (MEME,

660 omegaMap), the specific location of those candidate sites in each gene (especially the single

661 candidate site in the known sperm-binding domain of each ZP gene; Fig. 1), and the distinctive

662 patterns of population genetic variation including strongly balanced polymorphisms (Table 4)

663 that were similar across all three genes (Fig. 2), all point toward selection acting on functional

664 variation involving these three genes and their epistatic interactions. 
These interpretations have several corollaries that could form the basis for further tests of

666

667

668

669

670

671

672

673

674

675

676

677

678

679

680

681

682

683

684

685

686

687

688

689

690

691

the hypothesis of coevolution among candidate sites in these three gamete-recognition genes under selection. The similar patterns of population genetic variation across the three genes suggest that both $Z P 3$ and $Z P 2$ coevolve with $C 4 B P A$, but the available data do not allow us to test the functional association between fertility and variation at the $Z P 3$ candidate site. Genotype and fertility data for all three candidate sites including $Z P 3315$ from large population samples are needed to test that expected association, and compare it to the effects of $Z P 236$ variation on fertility. Similarly, LD is expected to be strong between C4BPA 300 and both of the candidate sites in ZP genes, but the available data do not include all three candidate sites genotyped in the same large population samples.

The evidence for functional and evolutionary covariation between $\mathrm{ZP}$ genes and $C 4 B P A$ is also consistent with evidence that those ZP gene products interact as a complex in the zona pellucida. Purified ZP3 in solution forms a homodimer complex in which a pair of proteins binds in antiparallel orientation (Han et al. 2010), and this head-to-tail dimerization brings the Nterminal region of one protein into contact with the C-terminal region of the other (including the C-terminal sperm-binding domain of ZP3 that shows evidence of selection). Moreover, ZP3 expressed in the zona pellucida is hypothesized to form a heterodimer with ZP2 via the same antiparallel orientation of their $\mathrm{N}$ - and C-terminal regions (Darie et al. 2008; Monné and Jovine 2011; Clark 2013; Wassarman and Litscher 2013). Such ZP3-ZP2 heterodimers could form a single sperm-binding structure that includes both $Z P 3315$ and $Z P 236$, interacts with ZP-binding proteins on the sperm head (including C4BPA), and mediates coevolution among all three genes via compensatory changes (e.g., Hughes 2012) between $Z P 3$ and $Z P 2$. Additional structural information for ZP proteins and their polymeric organization (e.g., Han et al. 2010) could form the basis for testing the predicted functional significance of the three candidate sites under selection that were identified here.

Our functional interpretation of the genetic evidence for selection on these three human genes is based mainly on mouse studies. An important assumption of that interpretation is that 
692 the mouse and human genes are orthologous. There is little doubt that mouse $Z p 3$ and human $693 Z P 3$ (and mouse $Z p 2$ and human $Z P 2$ ) are orthologs, but orthology between mouse $Z p 3 r$ and 694 human $C 4 B P A$ is unlikely. The two genes have similar coding sequence organization (including 695 a series of 7 or 8 sushi domains), and occur in similar locations on chromosome 1 relative to 696 other genes in the RCA gene cluster; in the mouse genome, $Z p 3 r$ is adjacent to a $\mathrm{C} 4$ binding 697 protein gene (called $C 4 b p$ in mouse, and $C 4 B P A$ in humans), and to a $C 4 b p$-like pseudogene 698 (C4bp-ps1). However, in the rat genome this part of the RCA gene cluster includes three 699 functional genes: $Z p 3 r, C 4 b p a$, and $C 4 b p b$. These comparisons to the rat genome suggest that 700 mouse $C 4 b p$ is orthologous with rat $C 4 b p a$ and human $C 4 B P A ; C 4 b p b$ has become a pseudogene 701 in the mouse (C4bp-ps1); and that the rodent gene expressed in the sperm acrosomal vesicle $702(Z p 3 r)$ is not found in humans and may be descended from a gene-duplication event in a 703 common ancestor shared by mice, rats, and other rodents but not shared with humans, other 704 primates, or other mammalian lineages.

705 The possibility that mouse $Z p 3 r$ is not orthologous with human $C 4 B P A$ adds a 706 complication to our interpretation of the genetic evidence for selection on both sperm- and egg707 expressed genes in humans. A single positively-selected codon in $C 4 B P A(C 4 B P A 300$, 708 rs2075520) is in linkage disequilibrium with SNPs in both $Z P 3$ (Rohlfs, Weir \& Swanson 2010) 709 and ZP2 (Table 5); shows population genetic evidence of selection (Table 3); and shows 710 genealogical patterns of haplotype variation similar to both $Z P 3$ and $Z P 2$ (Fig. 2). We propose 711 that those patterns are the consequence of selection acting on functional interactions between 712 human C4BPA and ZP proteins at fertilization, similar to previously documented interactions 713 between ZP3R in the mouse sperm acrosome and ZP proteins in the mouse zona pellucida. That 714 proposal, and our analyses of human $C 4 B P A$ evolution (and coevolution with $Z P 3$ and $Z P 2$ ), is 715 not dependent on orthology between $C 4 B P A$ and $Z p 3 r$. However, functional studies of 716 expression of $C 4 B P A$ in human sperm, and of interactions between human C4BPA and ZP 717 proteins in the human egg coat at fertilization, are needed in order to more fully understand the 718 roles of those genes in both primate and rodent fertilization. 
719 Significance of coevolution among human fertilization genes

720 Molecular evidence for selection on balanced polymorphisms in human gamete-binding genes is

721 potentially important because it implicates sexual selection in the evolution of human fertility

722 and identifies possible causes of clinical infertility (e.g., Männikkö et al. 2005; Pökkylä et al.

723 2011). However, other possible sources of selection should also be considered. Expression of

$724 C 4 B P A$ is well known to be involved in regulation of the classical complement pathway in

725 human immunity (Müller-Eberhard and Miescher 1985; Krushkal, Kemper \& Gigli 1998), and is

726 implicated in immune responses to viral pathogens including HIV (Chang et al. 2012).

727 Numerous codons were identified as targets of positive selection in divergence of $C 4 B P A$ among

728 human and other primate species (Cagliani et al. 2016). Balanced polymorphisms may be

729 common among human genes involved in host-pathogen interactions (Leffler et al. 2013;

730 DeGiorgio, Lohmueller \& Nielsen 2014; Azevedo et al. 2015; Cagan et al. 2016). In this context, 731 environmental variation in selection by pathogens could favour multiple $C 4 B P A$ alleles (e.g., in

732 African and non-African populations) when $C 4 B P A$ alleles are expressed in the complement

733 pathway, while the same $C 4 B P A$ polymorphisms could lead to balanced polymorphisms in $Z P 3$

734 and $Z P 2$ caused by selection favouring egg-sperm allele or genotype matching when $C 4 B P A$

735 alleles are expressed in sperm. That hypothesis is consistent with recent evidence for genome-

736 wide positive selection on primate genes (including $C 4 B P A$ ) that encode proteins involved in

737 innate immunity (van der Lee et al. 2017). In that study, positive selection for nonsynonymous

738 substitutions among primate species was correlated with the accumulation of nonsynonymous

739 polymorphisms within the human lineage in the same genes (including polymorphisms in

$740 C 4 B P A$ ), and was interpreted as evidence of ongoing selection among humans driven by viruses

741 and other pathogens. Moreover, among the three candidate sites analyzed in our study, the

742 largest allele-frequency difference among population groups in the 1000 Genomes Phase 1 data

743 was found at C4BPA 300, which has a four-fold difference in the minor allele frequency (Table

744 4). That large difference is consistent with the possibility that the evolution of this three-gene

745 system may be driven in part by pleiotropic selection on other effects of C4BPA polymorphisms 
746 such as in innate immunity. How this pleiotropy might interact with selection on the effects of

$747 C 4 B P A$ in fertility and its coevolution with ZP genes is not known.

748 Candidate sites under selection may be ancient balanced polymorphisms

749 The hypothesis of coevolution based on balancing selection and allelic matching between male-

750 and female-expressed gamete-recognition genes (Rohlfs, Swanson \& Weir 2010) is also

751 consistent with limited data from the genomes of archaic humans in Eurasia, in which the

752 occurrence of these same polymorphisms at candidate sites under selection suggests that the

753 polymorphisms may be older than the origins of early modern humans in Africa. Among the

754 relatively low-coverage genomes for several Neanderthal individuals (Green et al. 2010) in the

755 UCSC genome browser, no sequence reads are mapped to the candidate sites in $C 4 B P A$

756 (rs4844573) or ZP3 (rs2906999); one of those genomes (Vi33.16) includes several sequence

757 reads that map to the candidate site in $Z P 2$ (rs2075520), and all of those reads include the

758 derived allele (ZP2 36 Gly). However, in the more recent high-coverage genome for a

759 Neanderthal individual (Prüfer et al. 2014), there is a mix of ancestral and derived alleles at these

760 sites: two ancestral C4BPA 300 alleles (a Thr-Thr homozygote); one ancestral and one derived

761 ZP3 315 allele (a Pro-Ser heterozygote); and two derived ZP2 36 alleles (a Gly-Gly

762 homozygote). Similarly, in the high-coverage genome for a Denisovan individual (Meyer et al.

763 2012), multiple reads map to all three of those candidate sites, and all reads encode the derived

764 allele for each site. The occurrence of both ancestral and derived alleles in archaic humans

765 outside of Africa is similar to variation at the same candidate sites in modern human genomes,

766 and is consistent with the hypothesis that these balanced polymorphisms have been maintained

767 for an evolutionarily long time, perhaps as long as the early-Pleistocene divergence between

768 archaic and modern humans (>500,000 years; see Kelso and Prüfer 2014). That pattern of shared

769 alleles between archaic and modern humans is similar to the pattern of shared $Z p 3$ and $Z p 2$

770 haplotypes between closely related mouse species (Turner and Hoekstra 2006) that were

771 interpreted as the products of balancing selection acting on long time scales and leading to trans- 
772 specific polymorphisms (Turner and Hoekstra 2008a,b). That pattern is also similar to (but not as

773 old as) trans-specific polymorphisms shared between extant humans and other extant great apes

774 that have been attributed to the effects of balancing selection (Azevedo et al. 2015). The parallel

775 patterns of balanced polymorphisms at all three genes studied here indicate possibly important

776 effects of selection on the expression and function of those gene products during human

777 fertilization.

\section{Acknowledgments}

779 Thanks to M. Elliot (University of Cambridge) for scripts and help in formatting data from the

7801000 Genomes Project; and to F. Breden and B. Crespi (Simon Fraser University) for advice and 781 comments.

\section{References}

7831000 Genomes Project Consortium. 2012. An integrated map of genetic variation from 1,092 784 human genomes. Nature 491:56-65.

785 Andersson M, Simmons LW. 2006. Sexual selection and mate choice. Trends in Ecology and 786 Evolution 21:296-302.

787 Andrés AM, Hubisz MJ, Indap A, Torgerson DG, Degenhardt JD, Boyko AR, Gutenkunst RN,

788 White TJ, Green Ed, Bustamante CD, Clark AG, Nielsen R. 2009. Targets of balancing 789 selection in the human genome. Molecular Biology and Evolution 26:2755-2764.

790 Anisimova M, Liberles DA. 2012. Detecting and understanding natural selection. In: Cannarozzi 791 GM, Schneider A, eds. Codon evolution. Oxford: Oxford University Press, 73-96.

792 Avella MA, Xiong B, Dean J. 2013. The molecular basis of gamete recognition in mice and 793 humans. Molecular Human Reproduction 19:279-289. 
794 Azevedo L, Serrano C, Amorim A, Cooper DN. 2015. Trans-species polymorphism in humans

795 and the great apes is generally maintained by balancing selection that modulates the host

796 immune response. Human Genomics 9:21.

797 Baibakov B, Boggs NA, Yauger B, Baibakov G, Dean J. 2012. Human sperm bind to the N-

798 terminal domain of ZP2 in humanized zonae pellucidae in transgenic mice. Journal of Cell

799 Biology 197:897-905.

800 Brockhurst MA, Chapman T, King KC, Mank JE, Paterson S, Hurst GDD. 2014. Running with

801 the Red Queen: the role of biotic conflicts in evolution. Proceedings of the Royal Society $B$ -

$802 \quad$ Biological Sciences 281:20141382.

803 Brown AHD. 1975. Sample sizes required to detect linkage disequilibrium between two or three

804 loci. Theoretical Population Biology 8:184-201.

805 Buffone MG, Zhuang T, Ord TS, Hui L, Moss SB, Gerton GL. 2008. Recombinant mouse sperm

806 ZP3-binding protein (ZP3R/sp56) forms a high order oligomer that binds eggs and inhibits

807 mouse fertilization in vitro. Journal of Biological Chemistry 283:12438-12445.

808 Burnett LA, Anderson DM, Rawls A, Bieber AL, Chandler DE. 2011. Mouse sperm exhibit

809 chemotaxis to allurin, a truncated member of the cysteine-rich secretory protein family.

810 Developmental Biology 360:318-328.

811 Buzbas EO, Joyce P, Rosenberg NA. 2011. Inference on the strength of balancing selection for

812 epistatically interacting loci. Theoretical Population Biology 79:102-113.

813 Cagan A, Theunert C, Laayouni H, Santpere G, Pybus M, Casals F, Kay K, Navarro A, Marques-

814 Bonet T, Bertranpetit J, Andrés AM. 2016. Natural selection in the great apes. Molecular

$815 \quad$ Biology and Evolution 33: 3268-3283.

816 Cagliani R, Forni D, Filippi G, Mozzi A, De Gioia L, Pontremoli C, Pozzoli U, Bresolin N,

817 Clerici, M, Sironi M. 2016. The mammalian complement system as an epitome of host-

818 pathogen genetic conflicts. Molecular Ecology 25:1324-1339. 
819 Chang H-H, Soderberg K, Skinner JA, Banchereau J, Chaussabel D, Haynes BF, Ramoni M,

820 Letvin NL. 2012. Transcriptional network predicts viral set point during acute HIV-1

821 infection. Journal of the American Medical Informatics Association 19:1103-1109.

822 Chen J, Litscher ES, Wassarman PM. 1998. Inactivation of the mouse sperm receptor, mZP3, by

823 site-directed mutagenesis of individual serine residues located at the combining site for sperm.

824 Proceedings of the National Academy of Sciences of the USA 95:6193-6197.

825 Clark GF. 2013. The role of carbohydrate recognition during human sperm-egg binding. Human

$826 \quad$ Reproduction 28:566-577.

827 Clark NL, Gasper J, Sekino M, Springer SA, Aquadro CF, Swanson WJ. 2009. Coevolution of

828 interacting fertilization proteins. PLOS Genetics 5:e1000570.

829 Claw KG, George RD, Swanson WJ. 2014. Detecting coevolution in mammalian sperm-egg

830 fusion proteins. Molecular Reproduction and Development 81:531-538.

831 Clement M, Posada D, Crandall K. 2000. TCS: a computer program to estimate gene

832 genealogies. Molecular Ecology 9:1657-1660.

833 Coop G, Pickrell JK, Novembre J, Kudaravalli S, Li J, Absher D, Myers RM, Cavalli-Sforza LL,

834 Feldman, MW, Pritchard JK. 2009. The role of geography in human adaptation. PLOS

$835 \quad$ Genetics 5:e1000500.

836 Coyne JA, Orr HA. 2004. Speciation. Sunderland, MA: Sinauer Associates.

837 Darie CC, Janssen WG, Litscher ES, Wassarman PM. 2008. Purified trout egg vitelline envelope 838 proteins VE $\beta$ and VE $\gamma$ polymerize into homomeric fibrils from dimers in vitro. Biochimica et 839 Biophysica Acta 1784:385-392.

840 de Villena FP-M, Rodríguez de Córdoba S. 1995. C4BPAL2: a second duplication of the C4BPA 841 gene in the human $R C A$ gene cluster. Immunogenetics 41:139-143.

842 DeGiorgio M, Lohmueller KE, Nielsen R. 2014. A model-based approach for identifying 843 signatures of ancient balancing selection in genetic data. PLOS Genetics 10:e1004561.

844 Delport W, Poon AFY, Frost SDW, Pond SLK. Datamonkey 2010: a suite of phylogenetic 845 analysis tools for evolutionary biology. Bioinformatics 26:2455-2457. 
846 Delves PJ, Lund T, Roitt IV. 2002. Antifertility vaccines. Trends in Immunology 23:213-219.

847 Dixson AF. 2009. Sexual selection and the origins of human mating systems. New York: Oxford $848 \quad$ University Press.

849 Egea R, Casillas S, Barbadilla A. 2008. Standard and generalized McDonald-Kreitman test: a

850 website to detect selection by comparing different classes of DNA sites. Nucleic Acids

$851 \quad$ Research 36:W157-W162.

852 Ermert D., Blom AM. 2016. C4b-binding protein: the good, the bad and the deadly. Novel

853 functions of an old friend. Immunology Letters 169: 82-92.

854 Fay JC, Wu CI. 2000. Hitchhiking under positive Darwinian selection. Genetics 155:1405-1413.

855 Foll M, Gaggiotti OE. 2008. A genome scan method to identify seleted loci appropriate for both

856 dominant and codominant markers: a Bayesian perspective. Genetics 180:977-993.

857 Forstmeier W, Wagenmakers E-J, Parker TH. 2017. Detecting and avoiding likely false-positive

858 findings - a practical guide. Biological Reviews 92:1941-1968.

859 Gavrilets S. 2014. Is sexual conflict an "engine of speciation”? Cold Spring Harbor Perspectives 860 in Biology 6:a017723.

861 Gelman A, Loken E. 2014. The statistical crisis in science. American Scientist 102:460-465.

862 Goldman N, Yang Z. 1994. A codon-based model of nucleotide substitution for protein-coding 863 DNA sequences. Molecular Biology and Evolution 11:725-736.

864 Good JM, Wiebe V, Albert FW, Burbano HA, Kircher M, Green RE, Halbwax M, Andre C, 865 Atencia R, Fischer A, Pääbo S. 2013. Comparative population genomics of the ejaculate in 866 humans and the great apes. Molecular Biology and Evolution 30:964-976.

867 Green RE, Krause J, Briggs AW, Maricic T, Stenzel U, Kircher M, Patterson N, Li H, Zhai W, 868 Fritz MH-Y, Hansen NF, Durand EY, Malaspinas A-S, Jensen JD, Marques-Bonet T, Alkan

869 C, Prüfer K, Meyer M, Burbano HA, Good JM, Schultz R, Aximu-Petri A, Butthof A, Höber

870 B, Höffner B, Siegemund M, Weihmann A, Nusbaum C, Lander ES, Russ C, Novod N, 871 Affourtit J, Egholm M, Verna C, Rudan P, Brajkovic D, Kucan Z, Gusic I, Doronichev VB, 872 Golovanova LV, Lalueza-Fox C, de la Rasilla M, Fortea J, Fosas A, Schmitz RW, Johnson 
873 PLF, Eichler EE, Falush D, Birney E, Mullikin JC, Slatkin M, Nielsen R, Kelso J, Lachmann

874 M, Reich D, Pääbo S. 2010. A draft sequence of the Neandertal genome. Science 328:710-

$875 \quad 722$.

876 Hamm D, Mautz BS, Wolfner MF, Aquadro CF, Swanson WJ. 2007 Evidence of amino acid

877 diversity-enhancing selection within humans and among primates at the candidate sperm-

878 receptor gene PKDREJ. American Journal of Human Genetics 81:44-52.

879 Han L, Monné M, Okumura H, Schwend T, Cherry AL, Flot D, Matsuda T, Jovine L. 2010.

880 Insights into egg coat assembly and egg-sperm interaction from the x-ray structure of full-

$881 \quad$ length ZP3. Cell 143:404-415.

882 Hart MW, Sunday JM, Popovic I, Learning KJ, and Konrad CM. 2014. Incipient speciation of

883 sea star populations by adaptive gamete recognition coevolution. Evolution 68:1294-1305.

884 Hillarp A, Wiklund H, Thern A, Dahlbäck. 1997. Molecular cloning of rat C4b binding protein

885 alpha- and beta-chains: structural and functional relationships among human, bovine, rabbit,

886 mouse, and rat proteins. The Journal of Immunology 158:1315-1323.

887 Hirohashi J, Kamei N, Kubo H, Sawada H, Matsumoto M, Hoshi M. 2008. Egg and sperm

888 recognition systems during fertilization. Development Growth and Differentiation 50:S221-

$889 \quad$ S238.

890 Hofer T, Ray N, Wegmann D, Excoffier L. 2009. Large allele frequency differences between

891 human continental groups are more likely to have occurred by drift during range expansions

892 than by selection. Annals of Human Genetics 73:95-108.

893 Holsinger KE, Weir BS. 2009. Genetics in geographically structured populations: defining,

894 estimating and interpreting $F_{\mathrm{sт}}$. Nature Reviews Genetics 10:639-650.

895 Huang H-L, Lv C, Zhao Y-C, Li W, He X-M, Li P, Sha AG, Tian X, Papasian CJ, Deng HW, Lu

896 GX, Xiao HM. 2014. Mutant ZP1 in familial infertility. New England Journal of Medicine

$897 \quad 370: 1220-1226$.

898 Hughes AL. 2012. Amino acid sequence coevolution in the insect bursicon ligand-receptor

899 system. Molecular Phylogenetics and Evolution 63:617-624. 
Kaj I, Mugal CF. 2016. The non-equilibrium allele frequency spectrum in a Poisson random field framework. Theoretical Population Biology 111:51-64.

902 Kelso J, Prüfer K. 2014. Ancient humans and the origin of modern humans. Current Opinion in $903 \quad$ Genetics \& Development 29:133-138.

904 Kent WJ, Sugnet CW, Furey TS, Roskin KM, Pringle TH, Zahler AM, Haussler D. 2002. The 905 human genome browser at UCSC. Genome Research 12:996-1006.

906 Key FM, Teixeira JC, de Filippo C, Andrés AM. 2014. Advantageous diversity maintained by 907 balancing selection in humans. Current Opinion in Genetics \& Development 29:45-51. 908 Kosakovsky Pond SL, Posada D, Gravenor MB, Woelk CH, Frost SDW. 2006. Automated 909 phylogenetic detection of recombination using a genetic algorithm. Molecular Biology and $910 \quad$ Evolution 23:1891-1901.

911 Kosova G, Abney M, Ober C. 2010. Heritability of reproductive fitness traits in a human 912 population. Proceedings of the National Academy of Sciences of the USA 107 Supplement $913 \quad 1:$ S1772-1778.

914 Kosova G, Pickrell JK, Kelley JL, McArdle PD, Shuldiner AR, Abney M, Ober C. 2010. The 915 CFTR Met 470 allele is associated with lower birth rates in fertile men from a population 916 isolate. PLOS Genetics 6:e1000974.

917 Kosova G, Scott NM, Niederberger C, Prins GS, Ober C. 2012. Genome-wide association study 918 identifies candidate genes for male fertility traits in humans. American Journal of Human 919 Genetics 90:950-961.

920 Krushkal J, Bat O, Gigli I. 2000. Evolutionary relationships among proteins encoded by the 921 regulator of complement activation gene cluster. Molecular Biology and Evolution 17:17189221730.

923 Krushkal J, Kemper C, Gigli I. 1998. Ancient origin of human complement factor H. Journal of $924 \quad$ Molecular Evolution 47:625-630.

925 Kryazhimskiy S, Plotkin JB. 2008. The population genetics of dN/dS. PLOS Genetics $926 \quad 4: \mathrm{e} 1000304$. 
927 Lessios HA. 2011. Speciation genes in free-spawning marine invertebrates. Integrative and 928 Comparative Biology 51:456-465.

929 Leffler EM, Gao Z, Pfeifer S, Ségurel L, Auton A, Venn O, Bowden R, Bontrop R, Wall JD,

930 Sella G, Donnelly P, McVean G, Przeworski M. 2013. Multiple instances of ancient balancing

931 selection shared between humans and chimpanzees. Science 339:1578-1582.

932 Letunic I, Doerks T, Bork P. 2015. SMART: recent updates, new developments and status in 933 2015. Nucleic Acids Research 43:D257-D260.

934 Levitan DR. 2012. Contemporary evolution of sea urchin gamete-recognition proteins:

935 experimental evidence of density-dependent gamete performance predicts shifts in allele 936 frequencies over time. Evolution 66:1722-1736.

937 Litscher ES, Williams Z, Wassarman PM. 2009. Zona pellucida glycoprotein ZP3 and 938 fertilization in mammals. Molecular Reproduction and Development 76:933-941.

939 Lou YH, Ang J, Thai H, McElveen F, Tung KSK. 1995. A zona pellucida 3 peptide vaccine 940 induces antibodies and reversible infertility without ovarian pathology. Journal of 941 Immunology 155:2715-2720.

942 Männikkö M, Törmälä R-M, Tuuri T, Haltia A, Martikainen H, Ala-Kokko L, Tapanainen JS, 943 Lakkakorpi JT. 2005. Association between sequence variation in genes encoding human zona 944 pellucida glycoproteins and fertilization failure in IVF. Human Reproduction 20:1578-1585. 945 Mayilyan, KR. 2012. Complement genetics, deficiencies, and disease associations. Protein \& $946 \quad$ Cell 3:487-496.

947 McDonald JH, Kreitman M. 1991. Adaptive protein evolution at the Adh locus in Drosophila. $948 \quad$ Nature 351:652-654.

949 Meeusen ENT, Walker J, Peters A, Pastoret PP, and Jungersen G. 2007. Current status of 950 veterinary vaccines. Clinical Microbiology Reviews 20:489-510.

951 Meslin C, Mugnier S, Callebaut I, Laurin M, Pascal G, Poupon A, Goudet G, Monget P. 2012.

952 Evolution of genes involved in gamete interaction: evidence for positive selection, 953 duplications, and losses in vertebrates. PLOS One 7:e44548. 
954 Meyer M, Kircher M, Gansauge M-T, Li H, Racimo F, Mallick S, Schraiber JG, Jay F, Prüfer K,

955 de Filippo C, Sudmant PH, Alkan C, Fu QM, Do R, Rohland N, Tandon A, Siebauer M,

956 Green RE, Bryc K, Briggs AW, Stenzel U, Dabney J, Shendure J, Kitzman J, Hammer MF,

957 Shunkov MV, Derevianko AP, Patterson N, Andres AM, Eichler EE, Slatkin M, Reich D,

958 Kelso J, Pääbo S. 2012. A high-coverage genome sequence from an archaic Denisovan

959 individual. Science 338:222-226.

960 Monné M, Jovine L. 2011. A structural view of egg coat architecture and function in

961 fertilization. Biology of Reproduction 85:661-669.

962 Morgan CC, Loughran NB, Walsh TA, Harrison AJ, O’Connell MJ. 2010. Positive selection

963 neighboring functionally essential sites and disease-implicated regions of mammalian

964 reproductive proteins. BMC Evolutionary Biology 10:39.

965 Morgan CC, Loughran NB, Walsh TA, Harrison AJ, O’Connell MJ. 2017. Erratum to: Positive

966 selection neighboring functionally essential sites and disease-implicated regions of

967 mammalian reproductive proteins. BMC Evolutionary Biology 17:170.

968 Mugal CF, Wolf JBW, Kaj I. 2014. Why time matters: codon evolution and the temporal

969 dynamics of dN/dS. Molecular Biology and Evolution 31:212-231.

970 Müller-Eberhard HJ, Miescher PA, eds. 1985. Complement. New York: Springer-Verlag.

971 Muro Y, Buffone MG, Okabe M, Gerton GL. 2012. Function of the acrosomal matrix: zona

972 pellucida 3 receptor $(\mathrm{ZP} 3 \mathrm{R} / \mathrm{sp56})$ is not essential for mouse fertilization. Biology of

973 Reproduction 86:1-6.

974 Murrell B, Wertheim JO, Moola S, Weighill T, Scheffler K, Pond SLK. 2012. Detecting

975 individual sites subject to episodic diversifying selection. PLOS Genetics 8:e1002764.

976 Muse SV, Gaut BS. 1994. A likelihood approach for comparing synonymous and

977 nonsynonymous nucleotide substitution rates, with application to the chloroplast genome.

$978 \quad$ Molecular Biology and Evolution 11:715-724.

979 Nielsen R, Williamson S, Kim Y, Hubisz MJ, Clark AG, Bustamante C. 2005. Genomic scans

980 for selective sweeps using SNP data. Genome Research 15:1566-1575. 
981 Ostrowski EA, Shen Y, Tian X, Sucgang R, Jiang H, Qu J, Katoh-Kurasawa M, Brock DA, Dinh

982 C, Lara-Garduno F, Lee SL, Kovar CL, Dinh HH, Korchina V, Jackson L, Patil S, Han Y,

983 Chaboub L, Shaulsky G, Muzny DM, Worley KC, Gibbs RA, Richards S, Kuspa A,

984 Strassmann JE, Queller DC. 2015. Genomic signatures of cooperation and conflict in the

$985 \quad$ social amoeba. Current Biology 25:1661-1665.

986 Palumbi SR. 1999. All males are not created equal: fertility differences depend on gamete

987 recognition polymorphisms in sea urchins. Proceedings of the National Academy of Sciences

988 of the USA 96:12632-12637.

989 Palumbi SR. 2009. Speciation and the evolution of gamete recognition genes: pattern and

$990 \quad$ process. Heredity 102:66-76.

991 Patiño S, Keever CC, Sunday JM, Popovic I, Byrne M, Hart MW. 2016. Sperm bindin

992 divergence under sexual selection and concerted evolution in sea stars. Molecular Biology and

993 Evolution 33:1988-2001.

994 Pökkylä R-M, Lakkakorpi JT, Nuojua-Huttunen SH, Tapanainen JS. 2011. Sequence variations

995 in human $Z P$ genes as potential modifiers of zona pellucida architecture. Fertility and

$996 \quad$ Sterility 95:2669-2672.

997 Popovic I, Marko PB, Wares JP, Hart MW. 2014. Selection and demographic history shape the

998 molecular evolution of the gamete compatibility protein bindin in Pisaster sea stars. Ecology

$999 \quad$ and Evolution 4:1567-1588.

1000 Prüfer K, Racimo F, Patterson N, Jay F, Sankararaman S, Sawyer S, Heinze A, Renaud G,

1001 Sudmant PH, de Filippo C, Li H, Mallick S, Dannemann M, Fu Q, Kircher M, Kuhlwilm M,

1002 Lachmann M, Meyer M, Ongyerth M, Siebauer M, Theunert C, Tandon A, Moorjani P,

1003 Pickrell J, Mullikin JC, Vohr SH, Green RE, Hellman I, Johnson PLF, Blanche H, Cann H,

1004 Kitzman JO, Shendure J, Eichler EE, Lein ES, Bakken TE, Golovanova LV, Doronichev VB,

1005 Shunkov MV, Derevianko AP, Viola B, Slatkin M, Reich D, Kelso J, Pääbo S. 2014. The

1006 complete genome sequence of a Neanderthal from the Altai Mountains. Nature 505:43-49. 
1007 Puts DA. 2010. Beauty and the beast: mechanisms of sexual selection in humans. Evolution and $1008 \quad$ Human Behavior 31:157-175.

1009 Pybus M, Dall'Olio GM, Luisi P, Uzkudun M, Carreno-Torres A, Pavlidis P, Laayouni H, 1010 Bertranpetit J, Engelken J. 2014. 1000 Genomes Selection Browser 1.0: a genome browser 1011 dedicated to signatures of natural selection in modern humans. Nucleic Acids Research 1012 42:D903-D909.

1013 Qian W, Zhou H, Tang K. 2015. Recent coselection in human populations revealed by protein1014 protein interaction network. Genome Biology and Evolution 7:136-153.

1015 Rambaut A. 2002. Se-Al sequence alignment editor v2.0 software. Oxford: Department of 1016 Zoology, University of Oxford.

1017 Raymond M, Rousset F. 1995. GENEPOP: population genetics software for exact tests and 1018 ecumenicism. Journal of Heredity 86:248-249.

1019 Rodríguez de Córdoba S, Perez-Blas M, Ramos-Ruiz R, Sánchez-Corral P, de Villena, FP-M, 1020 Rey-Campos J. 1994. The gene coding for the $\beta$-chain of C4b-binding protein $(C 4 B P B)$ ha 1021 become a pseudogene in the mouse. Genomics 21:501-509.

1022 Rohlfs RV, Swanson WJ, Weir BS. 2010. Detecting coevolution through allelic association 1023 between physically unlinked loci. American Journal of Human Genetics 86:674-685.

1024 Rozas J, Sánchez-DelBarrio JC, Messeguer X, Rozas R. 2003. DnaSP, DNA polymorphism 1025 analyses by the coalescent and other methods. Bioinformatics 19:2496-2497.

1026 Sabeti PC, Varilly P, Fry B, Lohmueller J, Hostetter E, Cotsapas C, Xie X, Byrne EH, McCarroll 1027 SA, Gaudet R, Schaffner SF, Lander ES, The International HapMap Consortium. 2007. 1028 Genome-wide detection and characterization of positive selection in human populations. $1029 \quad$ Nature 449:913-918.

1030 Schoville SD, Flowers JM, Burton RS. 2012. Diversifying selection underlies the origin of 1031 allozyme polymorphism at the phosphoglucose isomerase locus in Tigriopus californicus. 1032 PLOS ONE 7:e40035. 
1033 Simmons JP, Nelson LD, Simonsohn U. 2011. False-positive psychology: undisclosed flexibility

1034 in data collection and analysis allows presenting anything as significant. Psychological

$1035 \quad$ Science 22:1359-1366.

1036 Sinervo B, Svensson E. 2002. Correlational selection and the evolution of genomic architecture.

$1037 \quad$ Heredity 89:329-338.

1038 Single RM, Martin MP, Gao X, Meyer D, Yeager M, Kidd JR, Kidd KK, Carrington M. 2007.

1039 Global diversity and evidence for coevolution of KIR and HLA. Nature Genetics 39:1114-

$1040 \quad 1119$.

1041 Stajich JE, Hahn MW. 2005. Disentangling the effects of demography and selection in human

1042 history. Molecular Biology and Evolution 22:63-73.

1043 Sunday JM, Hart MW. 2013. Sea star populations diverge by positive selection at a sperm-egg

1044 compatibility locus. Ecology and Evolution 3:640-654.

1045 Swanson WJ, Vacquier VD. 2002. Reproductive protein evolution. Annual Review of Ecology

1046 and Systematics 33:161-179.

1047 Swanson WJ, Yang Z, Wolfner MF, Aquadro CF. 2001. Positive Darwinian selection drives the

1048 evolution of several female reproductive proteins in mammals. Proceedings of the National

1049 Academy of Sciences of the USA 98:2509-2514.

1050 Szpiech ZA, Hernandez RD. 2014. selscan: an efficient multithreaded program to perform EHH-

1051 based scans for positive selection. Molecular Biology and Evolution 31:2824-2827.

1052 Tajima F. 1989. Statistical method for testing the neutral mutation hypothesis by DNA

1053 polymorphism. Genetics 123:585-595.

1054 Tamura K, Peterson D, Peterson N, Stecher G, Nei M, Kumar S. 2011. MEGA5: molecular

1055 evolutionary genetics analysis using maximum likelihood, evolutionary distance, and

1056 maximum parsimony methods. Molecular Biology and Evolution 28:2731-2739.

1057 Turner LM, Hoekstra HE. 2006. Adaptive evolution of fertilization proteins within a genus:

1058 variation in ZP2 and ZP3 in deer mice (Peromyscus). Molecular Biology and Evolution

$1059 \quad 32: 1656-1669$. 
1060 Turner LM, Hoekstra HE. 2008a. Causes and consequences of the evolution of reproductive 1061 proteins. International Journal of Developmental Biology 52:769-780.

1062

1063 Turner LM, Hoekstra HE. 2008b. Reproductive protein evolution within and between species:

1064 maintenance of divergent ZP3 alleles in Peromyscus. Molecular Ecology 17:12616-12628.

1065 Vacquier VD, Swanson WJ, Lee YH. 1997. Positive Darwinian selection on two homologous

1066 fertilization proteins: what is the selective pressure driving their divergence? Journal of

1067 Molecular Evolution 44:S15-S22.

1068 Vahdati AR, Wagner A. 2016. Parallel or convergent evolution in human population genomic

1069 data revealed by genotype networks. BMC Evolutionary Biology 16:154.

1070 van der Lee R, Wiel L, van Dam TJP, Huynen MA. 2017. Genome-scale detection of positive

1071 selection in nine primates predicts human-virus evolutionary conflicts. Nucleic Acids

$1072 \quad$ Research 45:10634-10648.

1073 Vicens A, Roldan ERS. 2014. Coevolution of positively selected IZUMO1 and CD9 in rodents:

1074 Evidence of interaction between gamete fusion proteins? Biology of Reproduction 90:113.

1075 Vitti JJ, Grossman SR, Sabeti PC. 2013. Detecting natural selection in genomic data. Annual

1076 Review of Genetics 47:97-120.

1077 Voight BF, Kudaravalli S, Wen X, Pritchard JK. 2006. A map of recent positive selection in the 1078 human genome. PLOS Biology 5:e147.

1079 Wang H, Choi Y, Tayo B, Wang X, Morris N, Zhang X, Broeckel U, Hanis C, Kardia S, Redline

1080 S, Cooper RS, Tang H, Zhu X. 2017. Genome-wide survey in African Americans

1081 demonstrates potential epistasis of fitness in the human genome. Genetic Epidemiology

$1082 \quad 41: 122-135$.

1083 Wassarman PM, Litscher ES. 2013. Biogenesis of the mouse egg's extracellular coat, the zona 1084 pellucida. Current Topics in Developmental Biology 102:243-266.

1085 Wassarman PM. 2009. Mammalian fertilization: the strange case of sperm protein 56. BioEssays $1086 \quad 31: 153-158$.

1087 Wellcome Trust Case Control Consortium. 2007. Genome-wide association study of 14,000 
1088 cases of seven common diseases and 3,000 shared controls. Nature 447:661-678.

1089 Weir BS, Cockerham CC. 1984. Estimating F-statistics for the analysis of population structure. $1090 \quad$ Evolution 38:1358-1370.

1091 Wilburn DB, Swanson WJ. 2016. From molecules to mating: rapid evolution and biochemical 1092 studies of reproductive proteins. Journal of Proteomics 135:12-25.

1093 Williams Z, Litscher ES, Jovine L, Wassarman PM. 2006. Polypeptide encoded by mouse ZP3 1094 exon-7 is necessary and sufficient for binding of mouse sperm in vitro. Journal of Cellular 1095 Physiology 207:30-39.

1096 Wilson DJ, McVean G. 2006. Estimating diversifying selection and functional constraint in the 1097 presence of recombination. Genetics 172:1411-1425.

1098 Yang Z. 2007. PAML 4: Phylogenetic analysis by maximum likelihood. Molecular Biology and 1099 Evolution 24:1586-1591.

1100 Zhong M, Zhang YW, Lange K, Fan RZ. 2011. A cross-population extended haplotype-based 1101 homozygosity score test to detect positive selection in genome-wide scans. Statistics and its $1102 \quad$ Interface 4:51-63.

1103 Zhou X, Stephens M. 2014. Efficient multivariate linear mixed model algorithms for genome$1104 \quad$ wide association studies. Nature Methods 11:407-409. 


\section{Figure 1 (on next page)}

Positive selection at individual codons in human gamete-recognition genes.

A single-population coalescent model of selection and recombination (omegaMap; Wilson and McVean 2006) identified the same candidate sites under positive selection that were identified by a branch-site model of codon evolution (MEME; Table 1). Values of $\omega$ were estimated using the sliding window method for alignments of haplotypes from the 1000 Genomes Phase 1 data for three gamete-recognition genes, including (A) the spermexpressed gene C4BPA, and the egg coat glycoprotein genes (B) ZP3 and (C) ZP2. Posterior probabilities of positive selection are shown for three candidate sites (see Table 1). 
A PeerJ
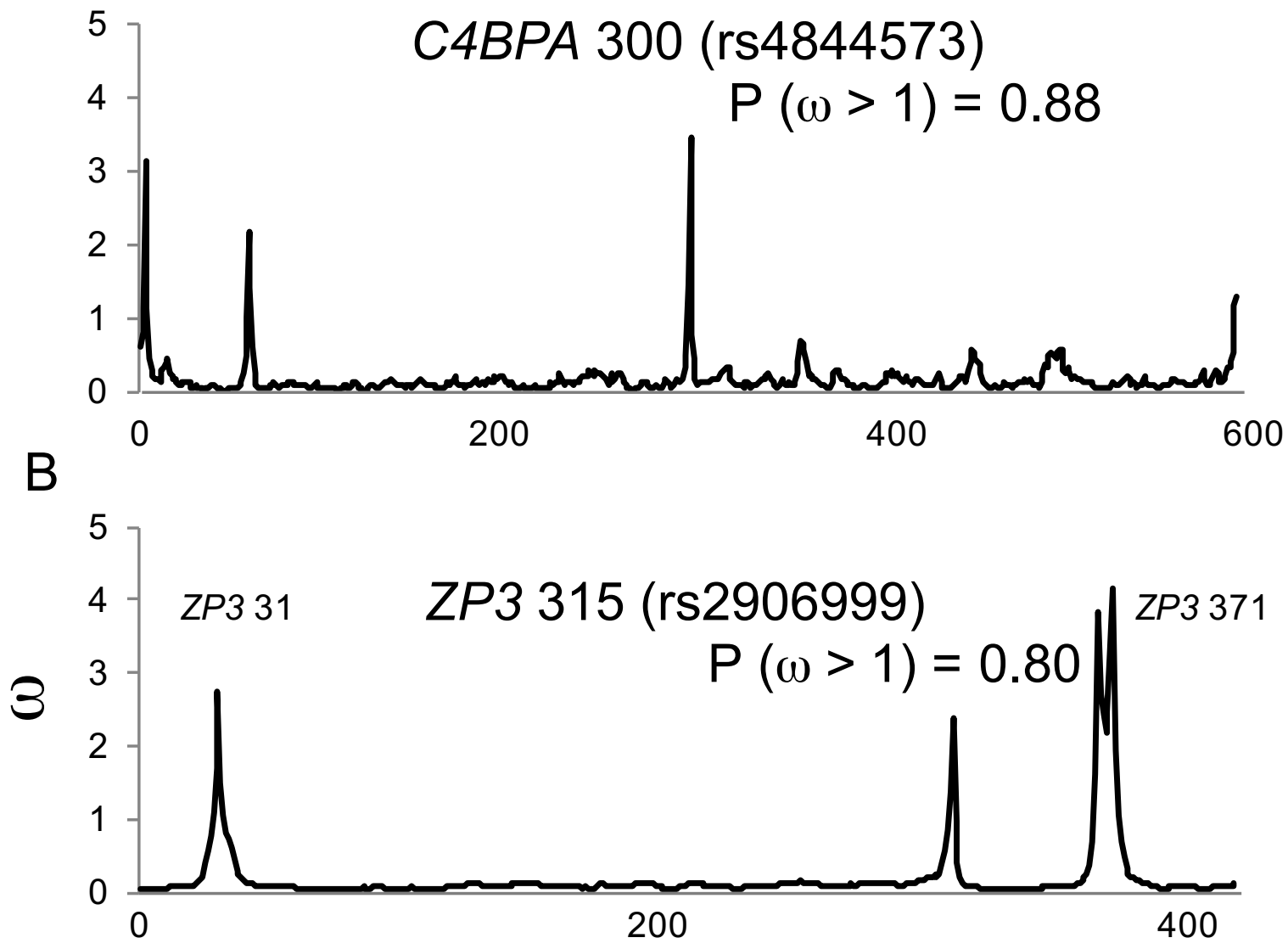

C

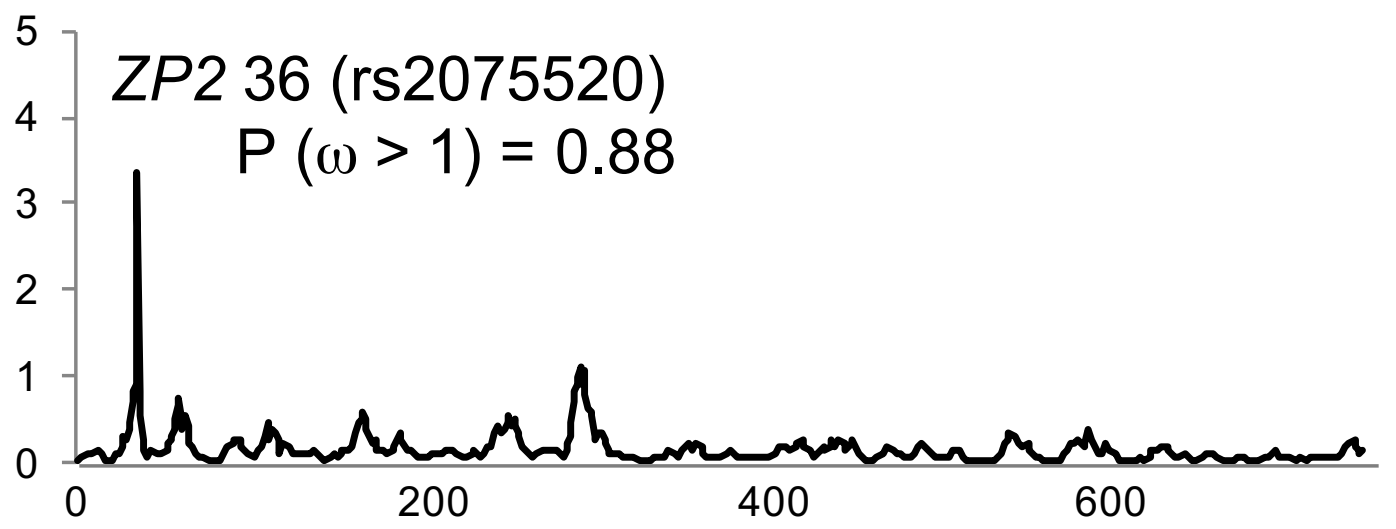

Peer) reviewing PDF | (2017:07:19056:1:1:REVIEW CeOzododon position 


\section{Figure 2 (on next page)}

Gene genealogies of human gamete-recognition genes.

Parsimony networks of relationships among haplotypes were similar for three genes including (A) the sperm-expressed gene C4BPA, and the egg coat glycoprotein genes (B) ZP3 and (C) $Z P 2$. Each diagram shows one of many most parsimonious genealogies, in which ambiguous relationships were represented by closed circuits in the parsimony network estimated by the TCS method (Clement, Posada \& Crandall 2000). For illustration, each closed circuit in the network was resolved to a single genealogy by favouring connections to more common haplotypes and pruning connections to less common haplotypes. Each square symbol represents a unique haplotype (44-65 per gene); the size of each square is proportional to relative frequency (1-300 gene copies out of 2184 total); large bold squares indicate common haplotypes that occurred $>300$ times. Lines show single nucleotide differences between haplotypes; red lines show nonsynonymous changes at one candidate site under selection in each gene (Table 2; Fig. 1). The ancestral amino acid state at each of those positivelyselected codons (see Table 2) is indicated for the common haplotype in that genealogy, and the direction of each substitution at the positively-selected site is indicated for each red line, including two parallel changes to the derived state (from proline to serine at ZP3 codon 315; from threonine to isoleucine at C4BPA codon 300) and one reversal to the ancestral state (from glycine to valine at ZP2 codon 36 ). For the two common haplotypes that differed by the non-synonymous polymorphism at the candidate site under selection, counts of that haplotype are shown for the four continental population groups from Europe (EUR), Asia $(A S N)$, the Americas (AMR), and Africa (AFR) in the 1000 Genomes Phase 1 release. 


\section{A $\mathrm{PeerJ}$ (4BPA (47 haplotypes)}

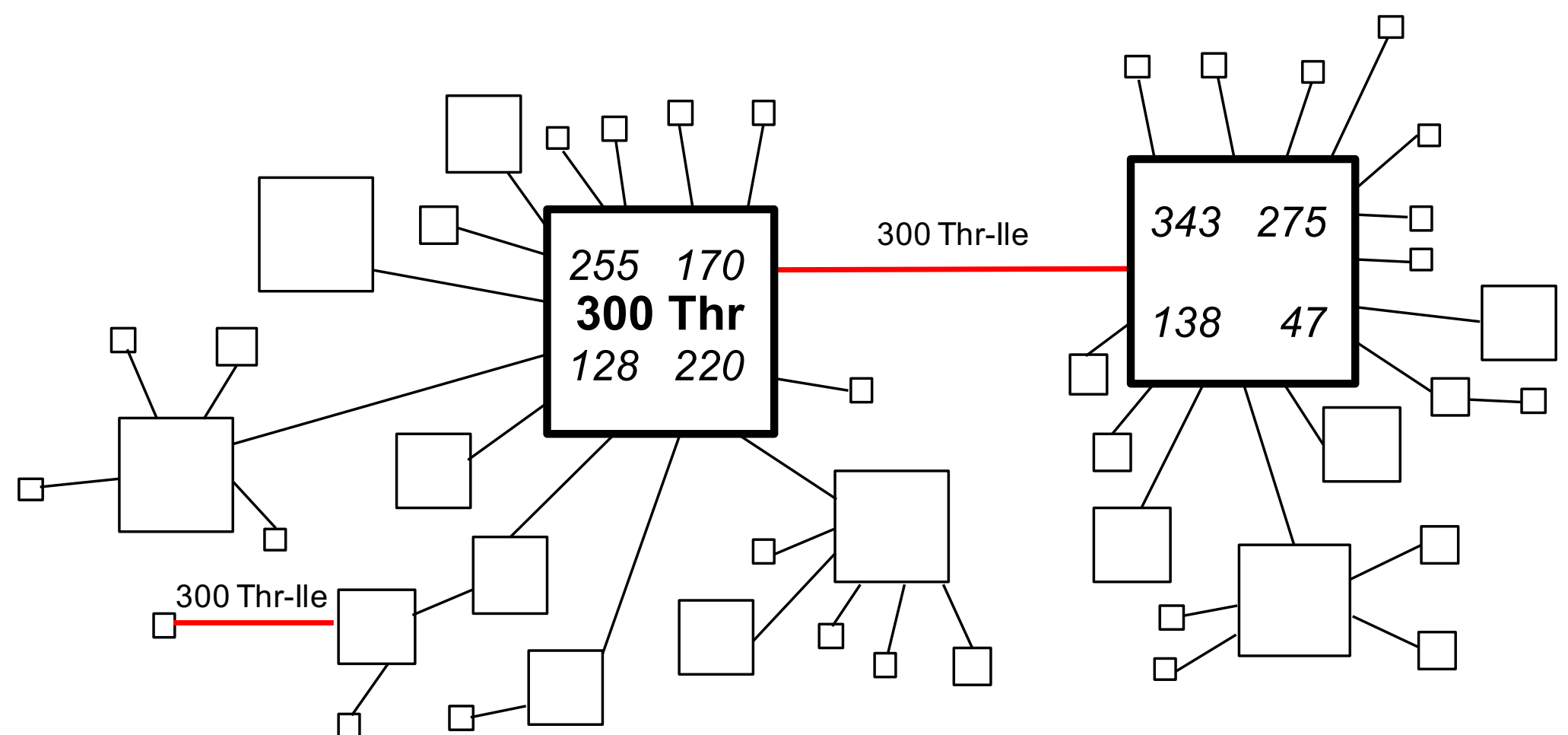

B ZP3 (65 haplotypes)
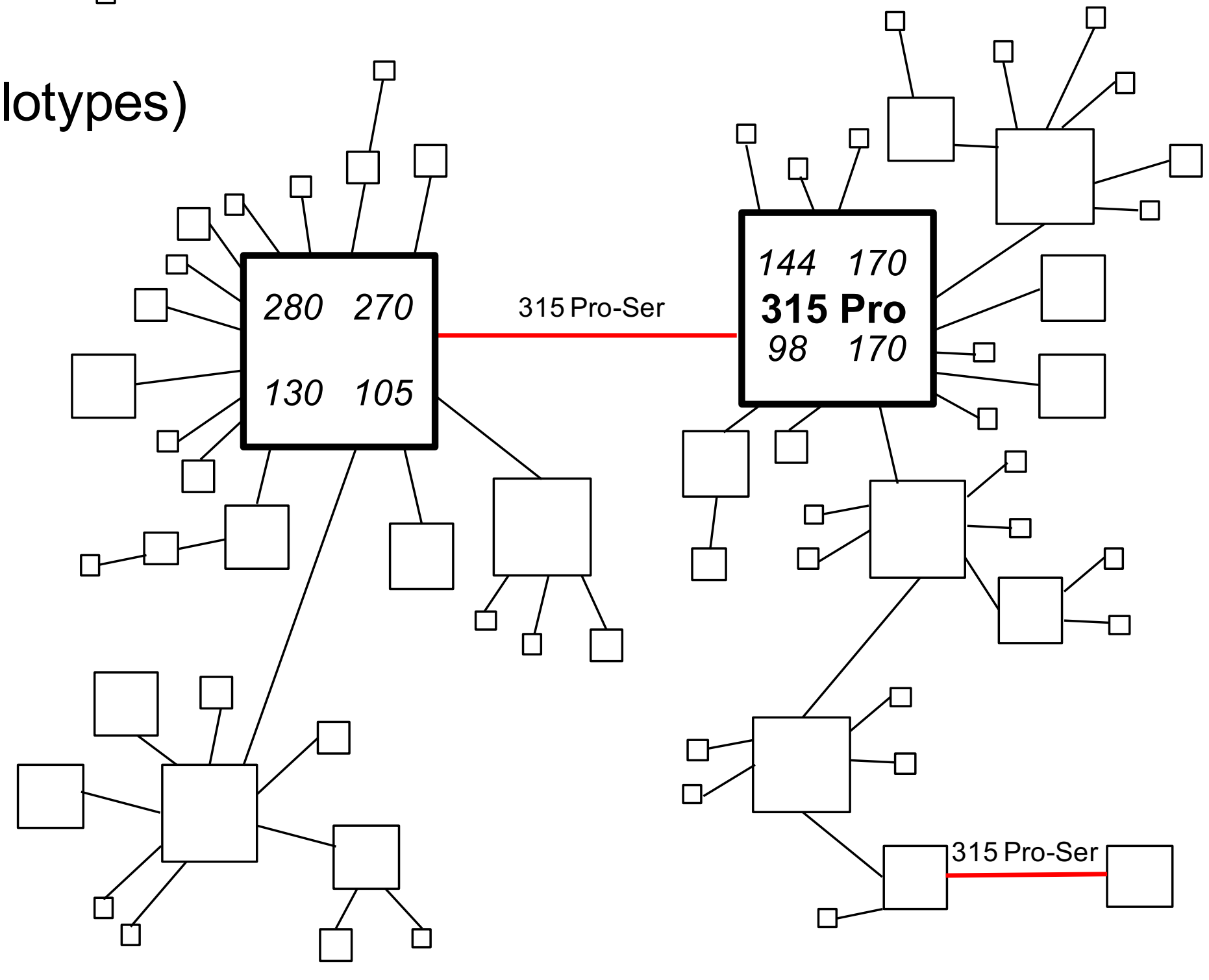

C ZP2 (44 haplotypes)

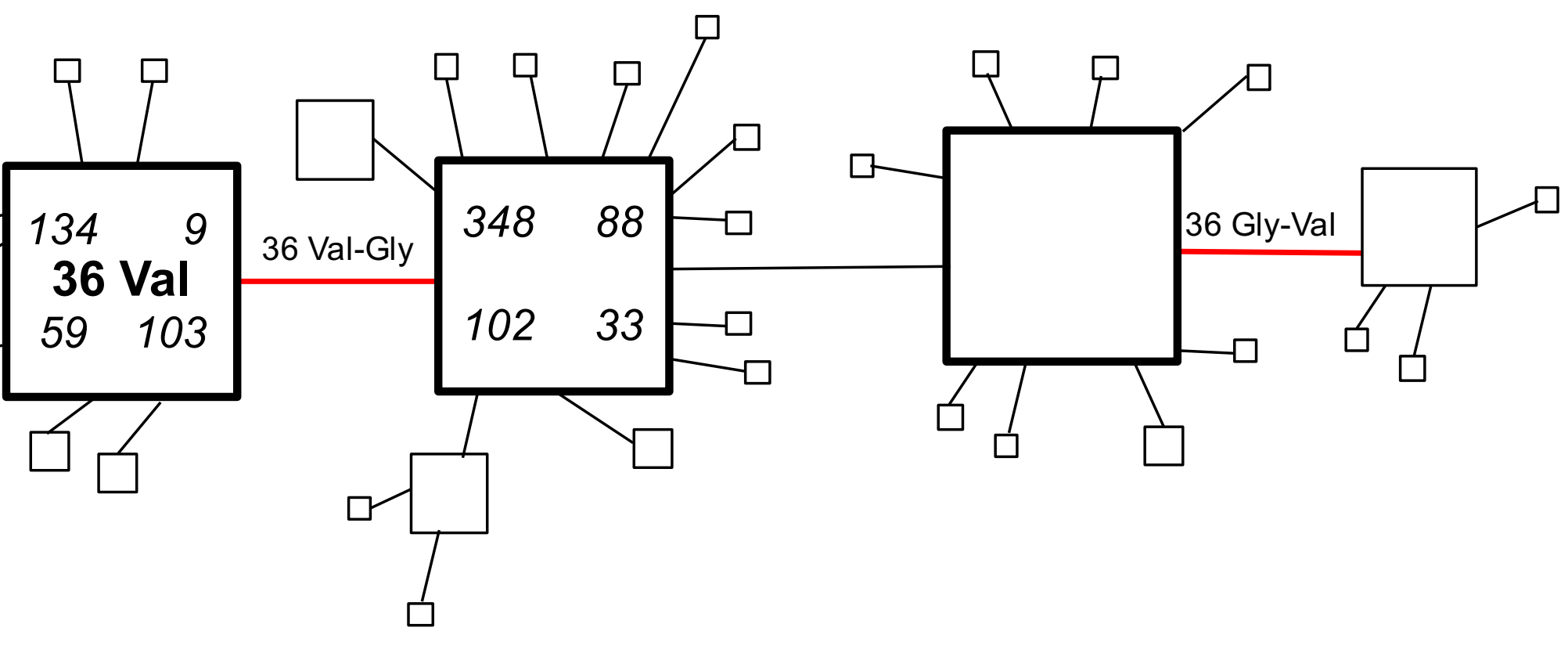




\section{Figure 3 (on next page)}

Fertility varies with genotype at two candidate sites under selection.

Hutterite families in which wives have more copies of the derived ZP2 36 Gly allele have fewer children (A), and families in which husbands have more copies of the derived C4BPA 300 lle allele have higher birth rates (B). Horizontal bars show means for each genotype class. Plotted values are residuals from linear model analyses (GEMMA) that account for three other covariates (wife's birth year, age at marriage, time from marriage to last birth); estimates of the statistical significance of those residual relationships also account for pedigree relatedness among families. 


\section{Table $\mathbf{1}$ (on next page)}

Site-specific parameter values estimated in a model of codon evolution fitted to alignments of human gamete-recognition genes.

Site-specific parameter values for mixed-effects models of evolution (MEME; Murrell et al. 2012) are shown for five codons in four analyses. Parameters include $\alpha$, the synonymous rate of change; $\beta$, the nonsynonymous rate at that site for lineages in the constrained class of codons with $\beta \leq \alpha$; and $\beta^{+}$, the unconstrained nonsynonymous rate (for codons in the positively-selected lineages of the gene tree). The probability of positive selection at each site $(P)$ is calculated from the likelihood ratio test of models with the constrained value of $\beta^{+}=\alpha$ versus the unconstrained $\beta^{+} ; q$ is the estimated false-discovery rate. Site-specific model parameter values are not shown for ZPI (na) because no candidate sites were assigned to the positively selected class with $\beta^{+}>\alpha$ at $P<0.10$. 


\begin{tabular}{|c|c|c|c|c|c|}
\hline \multirow[t]{2}{*}{ Codon } & \multicolumn{5}{|c|}{ MEME model parameter value } \\
\hline & $\alpha$ & $\beta-$ & $\beta+$ & $P$ & $q$ \\
\hline$C 4 P B A 300$, rs4844573 & 0 & 0 & 184 & 0.0079 & 1 \\
\hline ZP3 31, rs2286428 & 0 & 0 & 73.1 & 0.048 & 1 \\
\hline ZP3 315, rs2906999 & $5.2 \times 10^{-6}$ & $5.2 \times 10^{-6}$ & 186 & 0.012 & 1 \\
\hline ZP3 371, rs200481427 & 0 & 0 & 197 & 0.021 & 1 \\
\hline$Z P 236$, rs2075520 & $4.1 \times 10^{-9}$ & $4.1 \times 10^{-9}$ & 148 & 0.027 & 1 \\
\hline$Z P 1$ & na & na & na & all $P>0.10$ & \\
\hline
\end{tabular}




\section{Table 2 (on next page)}

Genomic organization of human gamete-recognition genes including candidate sites under selection.

Genome coordinates and gene structure are those in the UCSC genome browser. Ancestral alleles were identified by comparison to reference genes for other great apes. 


\begin{tabular}{|c|c|c|c|c|c|c|}
\hline Gene & $\begin{array}{l}\text { GRCh38/hg38 } \\
\text { Coordinates }\end{array}$ & $\begin{array}{l}\text { Number } \\
\text { of exons }\end{array}$ & $\begin{array}{l}\text { Number } \\
\text { of codons }\end{array}$ & $\begin{array}{l}\text { Candidate Site } \\
\text { Under Selection }\end{array}$ & $\begin{array}{l}\text { Ancestral } \\
\text { Allele }\end{array}$ & $\begin{array}{l}\text { Derived } \\
\text { Allele }\end{array}$ \\
\hline$C 4 B P A$ & $\begin{array}{l}\text { chr1:207,113,026- } \\
207,144,717\end{array}$ & 11 & 597 & $\begin{array}{l}\text { rs4844573 } \\
\text { chr1:207,131,555 } \\
\text { C4BPA } 300\end{array}$ & ACT Thr & ATT Ile \\
\hline$Z P 3$ & $\begin{array}{l}\operatorname{chr} 7: 76,424,965- \\
76,442,056\end{array}$ & 8 & 424 & $\begin{array}{l}\text { rs2906999 } \\
\text { chr7:76,440,494 } \\
\text { ZP3 } 315\end{array}$ & CCG Pro & TCG Ser \\
\hline$Z P 2$ & $\begin{array}{l}\text { chr16:21,197,480- } \\
21,211,547\end{array}$ & 19 & 745 & $\begin{array}{l}\text { rs2075520 } \\
\text { chr16:21,211,351 } \\
Z P 236\end{array}$ & GTG Val & GGG Gly \\
\hline$Z P 1$ & $\begin{array}{l}\text { Chr11:60,867,562- } \\
60,875,656\end{array}$ & 12 & 638 & none & & \\
\hline
\end{tabular}




\section{Table 3 (on next page)}

Population genetic tests of selection on candidate sites.

Results for three types of tests are shown based on (A) population differentiation, (B) linkage disequilibrium, or (C) the allele frequency spectrum. Each entry is the score or test statistic value (calculated using the 1000 Genomes Selection Browser v.1.0) for each candidate site under selection in a representative European (CEU), Asian (CHB), or African (YRI) population, or for each population pair (in pairwise $F_{\mathrm{ST}}$ and XP-EHH tests). Probability values are shown only for seven significant test results with $0.01<P<0.05$ based on the rank of each score relative to all other scores for other sites in a $2 \mathrm{Mbp}$ interval around the candidate site; all other test results were not significantly different from the null expectation $(P>0.05)$. Note that tests for the third candidate site (ZP3 315, rs2906999) could not be carried out using the 1000 Genomes Selection Browser v.1.0 because that site was not coded as variable in the 1000 Genomes Selection Browser database. Comparable tests for that missing site (using other methods or software) are given in the main text. 


\begin{tabular}{|c|c|c|c|c|}
\hline \multicolumn{2}{|c|}{ (A) Population differentiation } & \multicolumn{2}{|c|}{ Pairwise $F_{\mathrm{ST}}$} & \multirow[t]{2}{*}{ Global $F_{\mathrm{ST}}$} \\
\hline Gene, SNP & Population & CHB & YRI & \\
\hline \multirow[t]{2}{*}{ C4PBA 300, rs4844573 } & $\mathrm{CEU}$ & -0.005 & $0.323(P=0.030)$ & \multirow{2}{*}{$0.238(P=0.048)$} \\
\hline & $\mathrm{CHB}$ & & $0.342(P=0.036)$ & \\
\hline \multirow[t]{2}{*}{$Z P 2$ 36, rs2075520 } & CEU & 0.059 & $0.337(P=0.027)$ & \multirow{2}{*}{0.187} \\
\hline & $\mathrm{CHB}$ & & 0.142 & \\
\hline \multicolumn{2}{|c|}{ (B) Linkage disequilibrium } & \multicolumn{2}{|c|}{ XP-EHH } & \multirow[t]{2}{*}{ iHS } \\
\hline Gene, SNP & Population & $\mathrm{CHB}$ & YRI & \\
\hline \multirow[t]{3}{*}{ C4PBA 300, rs4844573 } & $\mathrm{CEU}$ & -0.564 & $1.935(P=0.045)$ & 0.594 \\
\hline & CHB & & $2.601(P=0.017)$ & 0.067 \\
\hline & YRI & & & $2.325(P=0.037)$ \\
\hline \multirow[t]{3}{*}{$Z P 2$ 36, rs2075520 } & $\mathrm{CEU}$ & 0.017 & -0.884 & 0.446 \\
\hline & $\mathrm{CHB}$ & & -0.488 & 0.140 \\
\hline & YRI & & & 1.129 \\
\hline \multicolumn{2}{|c|}{ (C) Allele frequency spectrum } & \multirow[t]{2}{*}{ Tajima's $D$} & \multirow[t]{2}{*}{ CLR } & \multirow[t]{2}{*}{ Fay \& Wu's $H$} \\
\hline Gene, SNP & Population & & & \\
\hline \multirow[t]{3}{*}{$C 4 P B A 300$, rs 4844573} & $\mathrm{CEU}$ & 0.716 & 0.496 & -10.432 \\
\hline & CHB & 1.491 & 0.152 & -4.853 \\
\hline & YRI & 0.383 & 0.340 & 3.263 \\
\hline \multirow[t]{3}{*}{$Z P 236$, rs2075520 } & $\mathrm{CEU}$ & 1.084 & 0.621 & 4.754 \\
\hline & $\mathrm{CHB}$ & 1.897 & 0.000 & -4.736 \\
\hline & YRI & -0.063 & 1.301 & -2.453 \\
\hline
\end{tabular}




\section{Table 4 (on next page)}

Allele frequencies at candidate sites under selection in human gamete-recognition genes.

Entries on the left show frequencies for ancestral and derived alleles (ancestral alleles listed first) in four continental population groups in the 1000 Genomes Phase 1 release. Entries on the right show the minor allele frequency (MAF) summed over all populations, and the number of nonsynonymous SNPs in a 90.3 Mbp interval on the same chromosome that had a MAF greater than or equal to the candidate site (relative to $\mathrm{N}$, the total number of nonsynonymous SNPS in parentheses). The significance of the MAF is characterized by $P$ (the proportion of Nwith a MAF greater than or equal to the MAF for the candidate site). 


\begin{tabular}{|c|c|c|c|c|c|c|c|c|}
\hline \multicolumn{2}{|c|}{ Candidate site } & \multicolumn{4}{|c|}{ Population group } & \multirow{2}{*}{$\begin{array}{l}\text { MAF } \\
0467\end{array}$} & \multirow{3}{*}{\begin{tabular}{|l} 
SNPs $(\boldsymbol{N})$ \\
$\geq$ MAF \\
$19(1408)$
\end{tabular}} & \multirow{3}{*}{$\begin{array}{l}\boldsymbol{P} \\
\\
0.013\end{array}$} \\
\hline & & \multirow{2}{*}{$\begin{array}{l}\text { Africa } \\
0.815\end{array}$} & \multirow{2}{*}{$\begin{array}{l}\text { Americas } \\
0.431\end{array}$} & \multirow{2}{*}{$\begin{array}{c}\text { Asia } \\
0.327\end{array}$} & \multirow{2}{*}{$\begin{array}{l}\text { Europe } \\
0.367\end{array}$} & & & \\
\hline $\begin{array}{l}C 4 B P A 300, \\
\text { rs4844573 }\end{array}$ & $\begin{array}{l}\text { ACT } \\
\text { Thr }\end{array}$ & & & & & 0.467 & & \\
\hline & $\begin{array}{l}\text { ATT } \\
\text { Ile }\end{array}$ & 0.185 & 0.569 & 0.673 & 0.633 & & & \\
\hline \multirow[t]{2}{*}{$\begin{array}{l}\text { ZP3 315, } \\
\text { rs2906999 }\end{array}$} & $\begin{array}{l}\text { CCG } \\
\text { Pro }\end{array}$ & 0.589 & 0.522 & 0.477 & 0.461 & & & \\
\hline & $\begin{array}{l}\text { TCG } \\
\text { Ser }\end{array}$ & 0.411 & 0.478 & 0.523 & 0.538 & 0.495 & $1(740)$ & 0.0014 \\
\hline \multirow[t]{2}{*}{$\begin{array}{l}Z P 236, \\
\text { rs2075520 }\end{array}$} & $\begin{array}{l}\text { GTG } \\
\text { Val }\end{array}$ & 0.648 & 0.356 & 0.460 & 0.273 & 0.420 & $61(1445)$ & 0.042 \\
\hline & $\begin{array}{l}\text { GGG } \\
\text { Gly }\end{array}$ & 0.352 & 0.644 & 0.540 & 0.727 & & & \\
\hline
\end{tabular}

1 


\section{Table 5 (on next page)}

Genotype associations between two candidate sites under selection in a founder population.

Each entry shows the observed (and expected) two-locus genotype frequencies in Hutterite families. Expected frequencies were calculated from genotype probabilities using the genotype association method in Rohlfs, Swanson \& Weir (2010). 


\begin{tabular}{|l|l|l|l|l|}
\hline ZP2 36 & \multicolumn{4}{|l}{ C4BPA 300 } \\
\cline { 2 - 5 } & Thr/Thr & Thr/Ile & Ile/Ile & Total \\
\hline Val/Val & $9(20.5)$ & $41(35.5)$ & $20(13.9)$ & 70 \\
\hline Val/Gly & $151(138.7)$ & $242(240.0)$ & $80(94.2)$ & 473 \\
\hline Gly/Gly & $255(255.7)$ & $435(442.4)$ & $182(173.8)$ & 872 \\
\hline Total & 415 & 718 & 282 & 1415 \\
\hline
\end{tabular}

1 